\title{
The role of binaries in the enrichment of the early Galactic halo
}

\section{Carbon-enhanced metal-poor stars - CEMP-s stars}

\author{
T. T. Hansen ${ }^{1,2}$, J. Andersen ${ }^{3,4}$, B. Nordström ${ }^{3,4}$, T. C. Beers ${ }^{5}$, V. M. Placco ${ }^{5}$, J. Yoon ${ }^{5}$, and L. A. Buchhave ${ }^{6,7}$ \\ ${ }^{1}$ Landessternwarte, ZAH, Heidelberg University, Königstuhl 12, Heidelberg, 69117, Germany \\ 2 Observatories of the Carnegie Institution of Washington, 813 Santa Barbara St., Pasadena, CA 91101, USA \\ e-mail: thansen@carnegiescience.edu \\ 3 Dark Cosmology Centre, The Niels Bohr Institute, University of Copenhagen, Juliane Maries Vej 30, 2100 Copenhagen, Denmark \\ 4 Stellar Astrophysics Centre, Department of Physics and Astronomy, Aarhus University, 8000 Aarhus C, Denmark \\ 5 Department of Physics and JINA Center for the Evolution of the Elements, University of Notre Dame, Notre Dame, IN 46556, USA \\ ${ }^{6}$ Harvard-Smithsonian Center for Astrophysics, Cambridge, MA 02138, USA \\ 7 Centre for Star and Planet Formation, University of Copenhagen, 1350 Copenhagen, Denmark
}

Received 21 September 2015 / Accepted 22 December 2015

\section{ABSTRACT}

\begin{abstract}
Context. Detailed spectroscopic studies of metal-poor halo stars have highlighted the important role of carbon-enhanced metal-poor (CEMP) stars in understanding the early production and ejection of carbon in the Galaxy and in identifying the progenitors of the CEMP stars among the first stars formed after the Big Bang. Recent work has also classified the CEMP stars by absolute carbon abundance, $A(\mathrm{C})$, into high- and low-C bands, mostly populated by binary and single stars, respectively.

Aims. Our aim is to determine the frequency and orbital parameters of binary systems among the CEMP-s stars, which exhibit strong enhancements of neutron-capture elements associated with the $s$-process. This allows us to test whether local mass transfer from a binary companion is necessary and sufficient to explain their dramatic carbon excesses.

Methods. We have systematically monitored the radial velocities of a sample of 22 CEMP- $s$ stars for several years with $\sim$ monthly, high-resolution, low S/N échelle spectra obtained at the Nordic Optical Telescope (NOT) at La Palma, Spain. From these spectra, radial velocities with an accuracy of $\approx 100 \mathrm{~m} \mathrm{~s}^{-1}$ were determined by cross-correlation with optimised templates.

Results. Eighteen of the 22 stars exhibit clear orbital motion, yielding a binary frequency of $82 \pm 10 \%$, while four stars appear to be single $(18 \pm 10 \%)$. We thus confirm that the binary frequency of CEMP- $s$ stars is much higher than for normal metal-poor giants, but not $100 \%$ as previously claimed. Secure orbits are determined for eleven of the binaries and provisional orbits for six long-period systems $(P>3000$ days), and orbital circularisation timescales are discussed.

Conclusions. The conventional scenario of local mass transfer from a former asymptotic giant branch (AGB) binary companion does appear to account for the chemical composition of most CEMP-s stars. However, the excess of $\mathrm{C}$ and $s$-process elements in some single CEMP- $s$ stars was apparently transferred to their natal clouds by an external (distant) source. This finding has important implications for our understanding of carbon enrichment in the early Galactic halo and some high-redshift damped lyman alpha (DLA) systems, and of the mass loss from extremely metal-poor AGB stars.
\end{abstract}

Key words. Galaxy: formation - Galaxy: halo - stars: chemically peculiar - binaries: spectroscopic - ISM: structure

\section{Introduction}

Over the past few decades, large spectroscopic surveys have identified numerous very metal-poor (VMP; $[\mathrm{Fe} / \mathrm{H}]<-2.0$ ) and extremely metal-poor $(\mathrm{EMP} ; \mathrm{Fe} / \mathrm{H}]<-3.0)$ stars in the halo system of the Milky Way. High-resolution follow-up spectroscopy has also provided an increasingly detailed picture of the star-to-star elemental-abundance variations that constrain the early chemical evolution of the Galaxy (for reviews, see Beers \& Christlieb 2005; Ivezic et al. 2012; Frebel \& Norris 2015). The abundance patterns of individual chemically-peculiar stars that deviate markedly from those of the bulk of Population II stars can then be used to identify the nature of the progenitors and nucleosynthetic processes responsible for the production of their distinctive chemical signatures. Dilution of these signatures by later mixing with the interstellar medium (ISM) of the Galaxy ultimately establishes the mean abundance trends for relatively more metal-rich stars.
A key element in this context is carbon, which is found to be over-abundant in a large fraction of VMP stars ( $220 \%$ for $[\mathrm{Fe} / \mathrm{H}] \leq-2)$. Carbon-enhanced metal-poor (CEMP) stars were originally identified among the VMP and EMP stars discovered in the HK survey of Beers, Preston, \& Shectman (Beers et al. 1985, 1992) and the Hamburg/ESO survey of Christlieb and collaborators (Christlieb et al. 2008), and supplemented by a number of surveys since. The fraction of CEMP stars rises with decreasing metallicity (conventionally tracked by the iron abundance, $[\mathrm{Fe} / \mathrm{H}])$; hence they are of particular importance for studies of the early chemical evolution of the Galactic halo.

The CEMP stars comprise a number of subclasses (see Beers $\&$ Christlieb 2005). The best-populated of these are the CEMP- $s$ and CEMP-no stars, characterised by the presence or absence of enhancements in $s$-process elements in addition to their carbon enhancement. The great majority of the former can be accounted for by scenarios involving transfer of enriched material from a 
Table 1. Coordinates, photometry, and abundances for the CEMP- $s$ and CEMP- $r / s$ stars monitored for radial-velocity variation.

\begin{tabular}{|c|c|c|c|c|c|c|c|c|c|}
\hline Stellar ID & RA (J2000) & Dec (J2000) & $V$ & $B-V$ & $\operatorname{Ref}_{\text {Phot }}$ & {$[\mathrm{Fe} / \mathrm{H}]$} & {$[\mathrm{C} / \mathrm{Fe}]$} & {$[\mathrm{Ba} / \mathrm{Fe}]$} & $\operatorname{Ref}_{\text {Abund }}$ \\
\hline \multicolumn{10}{|c|}{ CEMP- $s$} \\
\hline HE 0002-1037 & $00: 05: 23$ & $-10: 20: 23$ & 13.70 & 0.48 & $\mathrm{a}$ & -3.75 & +3.19 & +1.67 & 1 \\
\hline HE $0111-1346$ & $01: 13: 47$ & $-13: 30: 50$ & 12.48 & 1.31 & $\mathrm{~b}$ & -1.91 & +1.70 & $<+2.32$ & 2,1 \\
\hline HE $0151-0341$ & $01: 53: 43$ & $-03: 27: 14$ & 13.36 & 1.14 & $\mathrm{~b}$ & -2.46 & +2.46 & +1.22 & 2,1 \\
\hline HE 0206-1916 & 02:09:20 & $-19: 01: 55$ & 14.00 & 1.13 & $\mathrm{~b}$ & -2.09 & +2.10 & +1.97 & 3 \\
\hline HE 0319-0215 & $03: 21: 46$ & $-02: 04: 34$ & 13.79 & 1.39 & $\mathrm{~b}$ & -2.30 & +2.00 & +0.52 & 1 \\
\hline HE $0430-1609^{*}$ & $04: 32: 51$ & $-16: 03: 39$ & 13.17 & 1.25 & $\mathrm{~b}$ & -3.00 & +1.14 & +1.62 & 1 \\
\hline HE $0441-0652$ & $04: 43: 30$ & $-06: 46: 54$ & 14.23 & 1.02 & $\mathrm{~b}$ & -2.47 & +1.38 & +1.11 & 3 \\
\hline HE $0507-1430$ & $05: 09: 17$ & $-16: 50: 05$ & 14.49 & 1.54 & $\mathrm{~b}$ & -2.40 & +2.60 & +1.30 & 4 \\
\hline HE $0507-1653$ & 05:10:08 & $-14: 26: 32$ & 12.51 & 1.13 & $\mathrm{~b}$ & -1.38 & +1.29 & +1.89 & 3 \\
\hline HE $0854+0151$ & $08: 57: 30$ & $+01: 39: 50$ & 14.98 & 0.92 & $\mathrm{~b}$ & -1.80 & +1.60 & +0.82 & 1 \\
\hline HE 0959-1424 & 10:02:04 & $-14: 39: 22$ & 13.37 & 0.60 & $\mathrm{~b}$ & -1.42 & +2.30 & +1.24 & 1 \\
\hline HE $1031-0020$ & $10: 34: 24$ & $-00: 36: 09$ & 11.87 & 0.74 & $\mathrm{a}$ & -2.81 & +1.58 & +1.55 & 8 \\
\hline HE $1045+0226$ & $10: 48: 03$ & $+02: 10: 47$ & 14.10 & 1.01 & $\mathrm{a}$ & -2.20 & +0.97 & +1.24 & 5 \\
\hline HE $1046-1352$ & $10: 48: 30$ & $-14: 08: 12$ & 14.71 & 0.68 & $\mathrm{~b}$ & -2.76 & +3.30 & +1.38 & 1 \\
\hline CS 30301-015 & 15:08:57 & $+02: 30: 19$ & 13.04 & 1.00 & $\mathrm{~b}$ & -2.64 & +1.60 & +1.45 & 7 \\
\hline HE $1523-1155$ & $15: 26: 41$ & $-12: 05: 43$ & 13.23 & 1.35 & $\mathrm{~b}$ & -2.15 & +1.86 & +1.72 & 3 \\
\hline HE 2201-0345 & $22: 03: 58$ & $-03: 30: 54$ & 14.31 & 1.18 & $\mathrm{~b}$ & -2.80 & +2.30 & +0.62 & 1 \\
\hline HE 2312-0758 & $23: 14: 55$ & $-07: 42: 32$ & 14.32 & 1.02 & $\mathrm{a}$ & -3.47 & +1.86 & +1.99 & 1 \\
\hline HE 2330-0555 & $23: 32: 55$ & $-05: 38: 50$ & 14.56 & 0.85 & $\mathrm{~b}$ & -2.78 & +2.09 & +1.22 & 3 \\
\hline \multicolumn{10}{|c|}{ CEMP- $r / s$} \\
\hline HE $0017+0055$ & $00: 20: 22$ & $+01: 12: 07$ & 11.46 & 1.53 & $\mathrm{~b}$ & -2.40 & +2.17 & $>+1.99$ & 10 \\
\hline HE $0039-2635^{* *}$ & $00: 41: 40$ & $-26: 18: 54$ & 12.22 & 1.12 & $\mathrm{~b}$ & -2.90 & +2.63 & +2.03 & 6 \\
\hline LP 624-44 & $16: 43: 14$ & $-01: 55: 30$ & 11.68 & 1.16 & $\mathrm{a}$ & -2.72 & +2.25 & +2.83 & 9 \\
\hline
\end{tabular}

Notes. $^{(*)}=$ LP 775-30; ${ }^{(* *)}=$ CS 29497-034.

References. Photometry: a) Henden et al. (2015), b) Beers et al. (2007a). Abundances: 1) This work; 2) Kennedy et al. (2011); 3 ) Aoki et al. (2007); 4) Beers et al. (2007b); 5) Cohen et al. (2013); 6) Barbuy et al. (2005); 7) Aoki et al. (2002a); 8) Cohen et al. (2006); 9) Aoki et al. (2002b); 10) Jorissen et al. (2016a).

binary companion that has passed through the asymptotic giantbranch (AGB) stage of evolution.

The origin of the latter has still not been identified with certainty, but their binary frequency is not higher than among metal-poor giants in general (see Paper II of this series, Hansen et al. 2016). As discussed there, a number of lines of evidence strongly suggest that the CEMP-no stars contain the nucleosynthesis products of the very first stars born in the Universe, i.e., that they are bona-fide second-generation stars. A third, less populated, subclass is the CEMP-r/s stars (which exhibit enhancements of both $r$-process and $s$-process elements in addition to that of carbon); their origin is presently poorly understood and needs further observational attention.

Lucatello et al. (2005) carried out a limited, multi-epoch, radial-velocity monitoring survey of $19 \mathrm{CEMP}-s$ stars, and by combining this survey with results from previous authors, argued that some $68 \%$ of the stars in their sample exhibited evidence for radial-velocity variation. Based on the Duquennoy \& Mayor (1991) distributions of orbital elements for solar-type dwarfs and their simulation of the sampling of phase space by the velocity windows covered in their observations, they concluded that the observations were compatible with $100 \%$ of CEMP-s stars being members of binary (or multiple) systems. A reanalysis of this sample, augmented with new data by Starkenburg et al. (2014), came to a similar conclusion. However, we emphasise that the size of the sample considered, and the range of periods that could be examined based on these data, is still relatively small.

In this series of papers, we present the results of an eight-year programme of precise radial-velocity monitoring and prompt, systematic follow-up of potentially variable objects, for larger samples of chemically-peculiar VMP and EMP stars than have heretofore received such close attention. Our goal is to perform a solid test of whether the distinctive abundance signatures of these objects can be accounted for by alteration of their birth chemistry by highly evolved binary companions.

Hansen et al. (2011) first showed that the enhancement of $r$-process elements observed in a small fraction (3-5\%) of VMP and EMP stars is not causally connected to membership in a binary system, this conclusion was confirmed and further strengthened in Paper I of this series (Hansen et al. 2015b). Paper II (Hansen et al. 2016) examined the same question for the class of CEMP-no stars and found that only $17 \pm 9 \%$ (4 of 24 ) of their programme stars were binaries; this percentage is identical to the binary frequency found in metal-poor red giants. The present Paper III addresses the extent to which binaries may play a role in the origin of CEMP- $s$ and CEMP- $r / s$ stars, using the same approach.

This paper is outlined as follows: Sect. 2 summarises the selection of our programme stars and briefly describes our observational strategy and the techniques employed. Results are presented in Sect. 3, and Sect. 4 describes the orbital properties of our binary programme stars. In Sect. 5, we discuss the constraints imposed by these results on the progenitors of CEMP- $s$ stars; a similar discussion for CEMP- $r / s$ stars is provided in Sect. 6. Section 7 discusses the significance of the single stars identified in our programme, and Section 8 presents our conclusions and perspectives on what can be learned from future spectroscopic results on CEMP- $s$ and CEMP- $r / s$ stars.

\section{Sample selection, observations, and analysis}

\subsection{Sample definition}

Our sample of stars is presented in Table 1, which lists their $V$ magnitudes, $B-V$ colours, and published $[\mathrm{Fe} / \mathrm{H}]$, $[\mathrm{C} / \mathrm{Fe}]$, and 
T. T. Hansen et al.: The role of binaries in the enrichment of the early Galactic halo. III.

Table 2. Barium and europium abundances for the potential CEMP- $r / s$ stars in the sample.

\begin{tabular}{lrrrl}
\hline \hline Stellar ID & {$[\mathrm{Ba} / \mathrm{Fe}]$} & {$[\mathrm{Eu} / \mathrm{Fe}]$} & {$[\mathrm{Ba} / \mathrm{Eu}]$} & Ref. \\
\hline HE 0017+0055 & $>+1.9$ & +2.3 & $>-0.4$ & Jorissen et al. (2016a) \\
HE 0039-2635 & +2.03 & +1.80 & +0.23 & Barbuy et al. (2005) \\
HE 1031-0020 & +1.55 & $<+0.82$ & $>+1.27$ & Cohen et al. (2013) \\
CS 30301-015 & +1.45 & +0.20 & +1.25 & Aoki et al. (2002a) \\
LP 625-44 & +2.83 & +1.72 & +1.11 & Aoki et al. (2002b) \\
\hline
\end{tabular}

$[\mathrm{Ba} / \mathrm{Fe}]$ abundances, either from the literature or determined as described below.

The majority of our programme stars are selected from the Hamburg/ESO survey of Christlieb and collaborators (HES; Christlieb et al. 2008) with the addition of one star from the HK survey, CS 30301-015. The CEMP star LP 625-44 was added since it is well studied, and previous authors have suggested that it might be a CEMP-r/s star. Two of the sample stars are rediscoveries; HE 0039-2635 of the HK survey star CS 29497-034, and HE 0430-1609 of the high proper-motion star LP 775-30.

For the stars in Table 1 labelled solely with a " 1 " in the final column ("this work"), the $[\mathrm{Fe} / \mathrm{H}]$ and $[\mathrm{C} / \mathrm{Fe}]$ abundances were determined from medium-resolution $(R \sim 2000)$ spectra, using the $n$-SSPP pipeline software (described in detail by Beers et al. 2014). These candidates were selected from the CEMP candidate lists of Placco et al. $(2010,2011)$ and earlier spectroscopic follow-up of HES candidates over the last $25 \mathrm{yr}$. These stars would clearly benefit from higher resolution spectroscopic abundance analyses.

Additionally, a number of stars in our programme had no abundance estimate (or upper limit) barium available in the literature; the $[\mathrm{Ba} / \mathrm{Fe}]$ abundance is required to make a confident assignment of a star into the CEMP- $s$ subclass. For these stars we have derived $\mathrm{Ba}$ abundances (or upper limits) from our co-added high-resolution spectra, following the procedure described in Paper II. This exercise clearly confirms the classification of all of these stars as CEMP- $s$ stars (see Table 1).

Four stars in our sample, HE 0039-2635, HE 1031-0020, CS 30301-015, and LP 625-44, have been suggested in the literature to be CEMP- $r / s$ stars: Carbon stars showing enhancement in both $r$ - and $s$-process elements $(0.0<[\mathrm{Ba} / \mathrm{Eu}]<+0.5$; Beers \& Christlieb 2005). Additionally, during the preparation of this paper, Jorissen et al. (2016a) discovered that HE 0017+0055 also has a very high Eu abundance. Table 2 lists the $\mathrm{Ba}$ and $\mathrm{Eu}$ abundances for all these stars, along with their $[\mathrm{Ba} / \mathrm{Eu}] \mathrm{ra}-$ tios. The very high Eu abundance of HE 0017+0055, combined with the lower limit on its $\mathrm{Ba}$ abundance, cause its $[\mathrm{Ba} / \mathrm{Eu}]$ ratio to fall below the above formal limit. In summary, only HE 0039-2635 fully qualifies as a CEMP- $r / s$ star; nevertheless, $\mathrm{Eu}$ is detected in the three other stars of the sample and should be accounted for in any formation scenarios of the CEMP-r/s stars, which are discussed more fully in Sect. 6.

In the remainder of this paper, we retain the CEMP-r $/ s$ label for HE 0039-2635, HE 0017+0055, and LP 625-44. However, because the $\mathrm{Eu}$ abundance of HE 1031-0020 is only an upper limit and CS 30301-015 has only modestly enhanced Eu, $[\mathrm{Eu} / \mathrm{Fe}]=+0.20$, we discuss these stars together with the other CEMP- $s$ stars.

\subsection{Observing strategy}

The key scientific goal of our project was to identify the single and binary stars in the sample and, if possible, determine the orbital periods and eccentricities with sufficient precision to understand the general properties of each class of stars. Accordingly, our observing strategy throughout the programme was to monitor the radial velocities of the sample stars regularly, precisely, and systematically in a homogeneous manner over a sufficiently long time span to detect any spectroscopic binaries among the stars, building on the examples of Duquennoy \& Mayor (1991) and Carney et al. (2003).

Maintaining a roughly monthly cadence in the observations was considered adequate for the expected long orbital periods. Aiming for a precision of the individual observations of $\sim 100 \mathrm{~m} \mathrm{~s}^{-1}$, and continuing the observations for up to 2900 days allowed us to detect orbital motion with very long periods. Moreover, the observations were reduced and the velocities inspected promptly after every observing night, so that any incipient variability could be detected and the observing cadence adapted as appropriate for each target.

As described in Paper I, this strategy enabled us to identify the star HE 1523-0901 as a very low-inclination binary despite its awkward period of 303 days and velocity semi-amplitude of only $0.35 \mathrm{~km} \mathrm{~s}^{-1}$. This is evidence that we are able to detect binary orbits of even very low amplitude securely, as also seen in the results listed in Table 4. However, observations of a given star were discontinued when our key scientific objectives had been reached; spending precious telescope time to achieve ultimate precision per se was not a priority beyond that point.

\subsection{Observations and data analysis}

Following the above strategy, the observations, reductions, and analysis procedures were the same as those of Papers I and II of this series. These papers provide more details; here we only give a short summary. The stars were observed with the FIES spectrograph at the $2.5 \mathrm{~m}$ Nordic Optical Telescope (NOT). The spectra cover a wavelength range of $3640 \AA$ to $7360 \AA$, at a resolving power of $R \approx 46000$ and average signal-to-noise ratio $(\mathrm{S} / \mathrm{N})$ of $\approx 10$. Background contamination was minimised by observing the stars in grey time, when the cross-correlation profile peaks of the stellar spectrum and any moonlight spectrum were well separated in velocity space.

Reductions and multi-order cross-correlations were performed with software developed by L. Buchhave. The template spectra employed for a given target were either the spectrum of the star with maximum signal ("Strongest"); a co-added spectrum of all the best spectra ("Co-add"); a synthetic spectrum consisting of delta functions at the solar wavelengths of the strongest stellar lines ("Delta"); or a co-added spectrum of a bright CEMP- $s$ star (HE 0507-1653) with a spectrum similar to that of the object.

Depending on the average quality of the spectra for a given star ( $\mathrm{S} / \mathrm{N}$ ratio, line density, and strengths), the individual spectra were cross-correlated against one of these templates. The "Strongest" or "Co-add" templates were usually preferred, as they provide a perfect match to the stellar spectrum and thus 
Table 3. Number of observations, adopted templates, mean heliocentric radial velocities and standard deviations, observed time spans, and variability criterion $P\left(\chi^{2}\right)$ for the sample stars.

\begin{tabular}{lrlrrrrc}
\hline \hline Stellar ID & $N_{\text {obs }}$ & Template & $\begin{array}{r}\mathrm{RV}_{\text {mean }} \\
\left(\mathrm{km} \mathrm{s}^{-1}\right)\end{array}$ & $\begin{array}{r}\sigma \\
\left(\mathrm{km} \mathrm{s}^{-1}\right)\end{array}$ & $\begin{array}{r}\Delta T \\
(\text { Days })\end{array}$ & $P\left(\chi^{2}\right)$ & Binary \\
\hline HE 0002-1037 & 10 & Co-add & -31.295 & 5.957 & 1066 & 0.000 & Yes \\
HE 0111-1346 & 9 & Strongest & +40.920 & 8.404 & 1044 & 0.000 & Yes \\
HE 0151-0341 & 11 & Co-add & -35.685 & 9.136 & 1012 & 0.000 & Yes \\
HE 0206-1916 & 9 & Co-add & -199.536 & 0.121 & 1044 & 0.233 & No \\
HE 0319-0215 & 16 & Co-add & -225.782 & 2.357 & 2207 & 0.000 & Yes \\
HE 0430-1609 & 16 & Co-add & +231.821 & 1.727 & 1184 & 0.000 & Yes \\
HE 0441-0652 & 16 & Co-add & -30.647 & 2.655 & 2371 & 0.000 & Yes \\
HE 0507-1430 & 11 & Strongest & +44.802 & 7.920 & 1064 & 0.000 & Yes \\
HE 0507-1653 & 15 & Co-add & +348.280 & 4.859 & 2124 & 0.000 & Yes \\
HE 0854+0151 & 15 & Co-add & +138.297 & 7.798 & 1757 & 0.000 & Yes \\
HE 0959-1424 & 17 & HE 0507-1653 & +343.379 & 0.655 & 2736 & 0.000 & Yes \\
HE 1031-0020 & 22 & Co-add & +68.660 & 1.157 & 2923 & 0.000 & Yes \\
HE 1045+0226 & 6 & HE 0507-1653 & +131.498 & 0.280 & 803 & 0.223 & No \\
CS 30301-015 & 18 & Co-add & +86.607 & 0.077 & 2234 & 0.883 & No \\
HE 1046-1352 & 12 & Strongest & +79.471 & 21.250 & 1812 & 0.000 & Yes \\
HE 1523-1155 & 9 & Co-add & -42.607 & 3.781 & 502 & 0.000 & Yes \\
HE 2201-0345 & 27 & Co-add & -55.927 & 3.525 & 2943 & 0.000 & Yes \\
HE 2312-0758 & 11 & Co-add & +32.981 & 3.176 & 1066 & 0.000 & Yes \\
HE 2330-0555 & 17 & Co-add & -235.124 & 0.231 & 2573 & 0.543 & No \\
\hline & \multicolumn{7}{c}{ CEMP-r/s } \\
\hline HE 0017+0055 & 28 & Strongest & -80.219 & 1.168 & 2943 & 0.000 & Yes \\
HE 0039-2635 & 2 & Strongest & -47.739 & 6.136 & 278 & 0.000 & Yes \\
LP 625-44 & 28 & Co-add & +35.036 & 3.348 & 2667 & 0.000 & Yes \\
\hline
\end{tabular}

allow us to include the largest number of spectral orders in the correlations and optimise the precision of the derived radial velocities. However, for some low-signal spectra it was not possible to use these two templates; a Co-add spectrum of a bright CEMP- $s$ star was then used.

The spectra of the CEMP- $s$ (and CEMP- $r / s$ ) stars are generally richer in strong lines than those of the $r$-process-enhanced and CEMP-no stars discussed in Papers I and II. Therefore, most stars discussed here could be correlated with the "Strongest" or "Co-add" templates, with the same template being used for all spectra of a given star. The typical accuracy of the resulting radial velocities is $1-200 \mathrm{~m} \mathrm{~s}^{-1}$.

Finally, seven selected radial-velocity standard stars were monitored on every observing night throughout this programme. They are listed in Table 2 of Paper I, which gives the derived mean heliocentric velocities and standard deviations. The mean difference of our measured velocities for these stars from their standard values is $73 \mathrm{~m} \mathrm{~s}^{-1}$ with a standard deviation per star of $69 \mathrm{~m} \mathrm{~s}^{-1}$, demonstrating that our results are not limited by the stability of the spectrograph.

\section{Results}

The results of our radial-velocity monitoring of the sample of CEMP- $s$ and CEMP- $r / s$ stars in Table 1 are summarised in Table 3, which lists the number of observations $\left(N_{\mathrm{obs}}\right)$ for each star, the cross-correlation template used, the resulting mean heliocentric radial velocity $\left(\mathrm{RV}_{\text {mean }}\right)$ and its standard deviation $(\sigma)$, the observed time span $(\Delta T)$, the probability that the velocity is constant $\left(P\left(\chi^{2}\right)\right)$, and our conclusion on whether the star is a binary. The individual radial-velocity observations and their associated internal errors, computed as described in Paper I, are given in Appendix A.
A few stars in our sample have radial velocities reported in the literature. The published measurements and total time spans covered for the four stars without significant radial-velocity variations are listed in Table B.1. However, as these data are few in number and exhibit offsets of up to $1 \mathrm{~km} \mathrm{~s}^{-1}$ between different sources that cannot be properly evaluated, we have not included them in our computations of $P\left(\chi^{2}\right)$ and the accompanying discussion. For the binary stars, the literature data are reviewed in Sect. 3.3 and included in the orbital solutions when found useful.

\subsection{Identifying the single and binary stars}

As in Paper II, we have assessed the binary status of each individual star by calculating the standard $\chi^{2}$ parameter for variability to evaluate the probability, $P\left(\chi^{2}\right)$, that the radial velocity is constant within the observational errors. A floor error or velocity jitter of $\sim 100 \mathrm{~m} \mathrm{~s}^{-1}$ has been added in quadrature to the internal error to account for sources of external errors, such as guiding or atmospheric dispersion and any intrinsic variability (discussed below). The resulting values of $P\left(\chi^{2}\right)$ are listed in Table 3, and demonstrate that at least 18 of our programme stars exhibit highly significant radial-velocity variations over the eight-year period of monitoring, in most cases clearly due to orbital motion.

However, the context in which the computed $P\left(\chi^{2}\right)$ values are discussed in this paper is the opposite of that in Papers I and II. There, the underlying hypothesis was that of a general population of constant-velocity (i.e., single) stars with a uniform statistical distribution of $P\left(\chi^{2}\right)$ values between 0.01 and 1.00 (see Nordström et al. 1997, Fig. 4; and Carney et al. 2003, Fig. 2). Here, the default expectation, following Lucatello et al. (2005) and Starkenburg et al. (2014), is that all CEMP-s (and CEMP-r/s) stars are binaries (i.e., the frequency is $100 \%$ ). 
T. T. Hansen et al.: The role of binaries in the enrichment of the early Galactic halo. III.

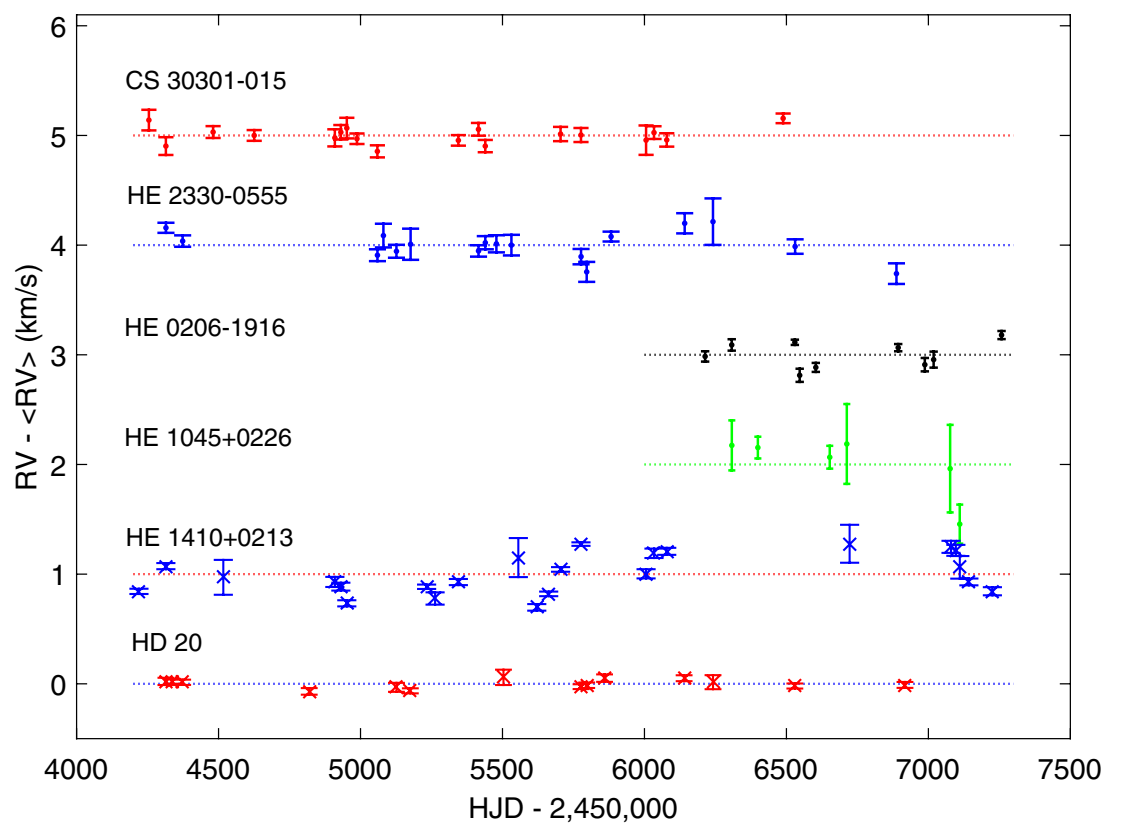

Fig. 1. Observed radial velocities for six constant stars from our project as functions of time, offset by $1 \mathrm{~km} \mathrm{~s}^{-1}$. Top to bottom: CS 30301-015, HE 2330-0555, HE 0206-1916, and HE 1045+0226 (this paper); HE 1410+0213 (CEMP-no, Paper II); and HD 20 ( $r$-I, Paper I).

Thus, the simulations of Lucatello et al. (2005) and Starkenburg et al. (2014) did in fact only allow them to conclude that the compilation of data available to them (establishing an observed frequency of true binaries of $68 \pm 11 \%$ ) was compatible with that hypothesis, but it did not prove that it was true. Additional assumptions in their simulations were that (i) the distributions of periods and other orbital parameters followed those derived by Duquennoy \& Mayor (1991) for solar-type dwarfs; and (ii) all significant radial-velocity variations were due to binary orbital motion.

Our finding that four stars in our sample exhibit no signs of any binary orbital motion appears to contradict that assumption, especially since 18 of our 22 stars $(82 \%)$ are well-established binaries with $P\left(\chi^{2}\right)$ values below $10^{-6}$ with secure or preliminary orbits for all but one, which is an even higher fraction of actual binaries than found by Lucatello et al. (2005).

Meanwhile, improvements in spectrograph design and radial-velocity precision has revealed low-amplitude, long-term velocity variations in essentially all normal and especially bright giants. Accordingly, the ability to detect binary (and exoplanet) orbital motions of even very low-amplitude and long-period binaries has not only improved owing to reduced observational errors, but it is also becoming increasingly limited by intrinsic variability in the target star itself. Moreover, not all potential binary companions are able to evolve past the AGB stage and transfer $s$-process enriched material to the surviving star, so only binary systems with companions of present-day mass in the white dwarf range of $0.5-1.4 M_{\odot}$ (Merle et al. 2016) need concern us here. We therefore discuss the binary status of our potentially single stars separately in the following section.

\subsection{The apparently single stars}

The four stars in Table 3 that are not established binaries all have $P\left(\chi^{2}\right)$ values well above the limit of $P\left(\chi^{2}\right)=0.05$, beyond which Carney et al. (2003) considered the stars to be safely single and demonstrated that the distribution of their $P\left(\chi^{2}\right)$ values is flat, as expected (see also Nordström et al. 1997, Fig. 4).
However, as also shown by Lucatello et al. (2005), a value of $Q\left(\chi^{2}\right)\left(=1-P\left(\chi^{2}\right)\right) \gtrsim 0.02$ actually indicates that most of them are in fact more likely to be variable than constant. This by itself does not prove that any such variation is necessarily caused by binary orbital motion; it must also exhibit a characteristic, significant, systematic, and well-sampled pattern (see e.g. Morbey \& Griffin 1987).

This is illustrated in Fig. 1, which compares the time histories of the velocities of the four single CEMP- $s$ stars in our sample with those of the single stars HE 1410+0213 (a CEMPno star, Paper II) and HD 20 (an $r$-I star, Paper I). Three of these stars (CS 30301-015, HE 0206-1916, and HD 20) have standard deviations of $\$ 100 \mathrm{~m} \mathrm{~s}^{-1}$ over their total periods of observation, and none of them exhibits any sign of orbital motion.

As discussed in detail in Paper II, HE 1410+0213 was initially suspected of showing orbital motion with a period near 341 days and semi-amplitude $\lesssim 300 \mathrm{~m} \mathrm{~s}^{-1}$, but this star did not continue this behaviour and eventually was judged to be a single, pulsating star. From the sample presented in this paper, HE $0017+0055$ is found to be a long-period binary (see Table 3), but it also exhibits an additional regular velocity variation of period $\approx 385$ days, $e \approx 0.15$, and semi-amplitude $K \approx 540 \mathrm{~m} \mathrm{~s}^{-1}$. Finally, in Paper I we found the highly $r$-process enhanced EMP star HE 1523-0901 to be a spectroscopic binary with a period of 303 days and semi-amplitude $350 \mathrm{~m} \mathrm{~s}^{-1}$.

Adopting these periods and amplitudes, modest orbital eccentricities, and setting $M_{1} \approx 0.8 M_{\odot}$ and $M_{2} \approx 0.5-1.4 M_{\odot}$ for the observed star and the presumed white dwarf companion, respectively, leads to orbital inclinations in the range $1.5^{\circ}-2^{\circ}$. Figure 1 suggests that any undiscovered binaries among our four "single" stars would have periods in the range 1000-10000 days or even longer and semi-amplitudes $\lesssim 100 \mathrm{~m} \mathrm{~s}^{-1}$, leading to similarly low orbital inclinations. Assuming a random distribution of orbits in space, the probability of finding even one such closely face-on orbit is $\approx 10^{-4}$, or less than $1 \%$ for our total sample of 63 stars. Having found HE 1523-0901 must then already be considered lucky; finding four such cases strains credulity.

Continued radial-velocity monitoring might still reveal orbital motion in one of our "constant" stars, notably in 
HE 1045+0226, but for now, we retain four as the most likely number of single stars, a fraction of $18 \pm 10 \%$. Thus, the great majority of the CEMP- $s$ stars are still in binaries, but exceptions to the local mass-transfer scenario for their origin do appear to exist, as is the case for the class of CEMP-no stars discussed in Paper II. Alternative scenarios are discussed in Sect. 7.

It is remarkable that two of our CEMP stars (HE 1410+0213 and HE 0017+0055) appear to exhibit near-periodic lowamplitude velocity variations of periods similar to those identified by Riebel et al. (2010) in the large Optical Gravitational Lensing Experiment (OGLE) data set of pulsating Large Magellanic Cloud (LMC) giants and C-rich AGB stars. Signatures of similar pulsations in another two CEMP stars are found in the sample of Jorissen et al. (2016b), but not among carbon-normal VMP/EMP $r$-process-enhanced stars. This suggests that the high molecular opacities of C-rich stellar atmospheres may be their source, but photometric confirmation of their existence and probable long periods in field stars is difficult from the ground.

Compounding this difficulty is the lack of reliable distances and absolute luminosities for isolated CEMP stars, and spectroscopic $\log g$ values are generally uncertain guides owing to inadequate resolution of these line-packed spectra. However, the impending precise trigonometric parallaxes and parallel uniform, precise, and well-sampled photometry from the Gaia mission should shortly put our understanding of the properties and evolution of these stars on a much safer footing.

\subsection{Binary stars}

Radial velocities have been published for a number of our stars that have been found to exhibit variable radial velocities. These data have been included in our discussion of certain or potential binaries in the following.

\section{CEMP-s stars:}

- HE 0111-1346 and HE 0507-1653 were also observed extensively by Jorissen et al. (2016b), with results agreeing with ours within the errors.

- HE 0430-1609 and HE 1523-1155 have suspiciously low rms errors for their orbital solutions. For HE 0430-1609, we can fit eccentric 2000-day and circular 4000-day orbits to our data, with identical rms errors of only $40 \mathrm{~m} \mathrm{~s}^{-1}$, but consider the latter more reliable (see end note). For HE 1523-1155, the velocities we derived from our observations from 2014 and 2015 plus the observation by Aoki et al. (2007) constrain the period accurately; in particular, much longer periods are excluded despite the significant orbital eccentricity of $e=0.3$. More observations of these two stars are needed in order to determine the final orbits with confidence.

- HE 0507-1653 and HE 1523-1155: Aoki et al. (2007) has reported single radial velocities for these two objects (353.0 $\mathrm{km} \mathrm{s}^{-1}$ and $-45.0 \mathrm{~km} \mathrm{~s}^{-1}$, respectively), which fit the orbital solutions derived from our own data; see Fig. 3.

- HE 0959-1424 undoubtedly has a variable velocity, as indicated by the values of $\sigma$ and $P\left(\chi^{2}\right)$ given in Table 3 . Figure 2 shows the measured radial velocities as a function of time. The slow, systematic velocity decrease suggests that HE 0959-1424 is a binary with a very long period, possibly of the order of 10000 days or even (much) longer, and a semi-amplitude of a few $\mathrm{km} \mathrm{s}^{-1}$. Low-amplitude, short-term velocity oscillations of the type seen in HE $0017+0055$ are not observed in this star.

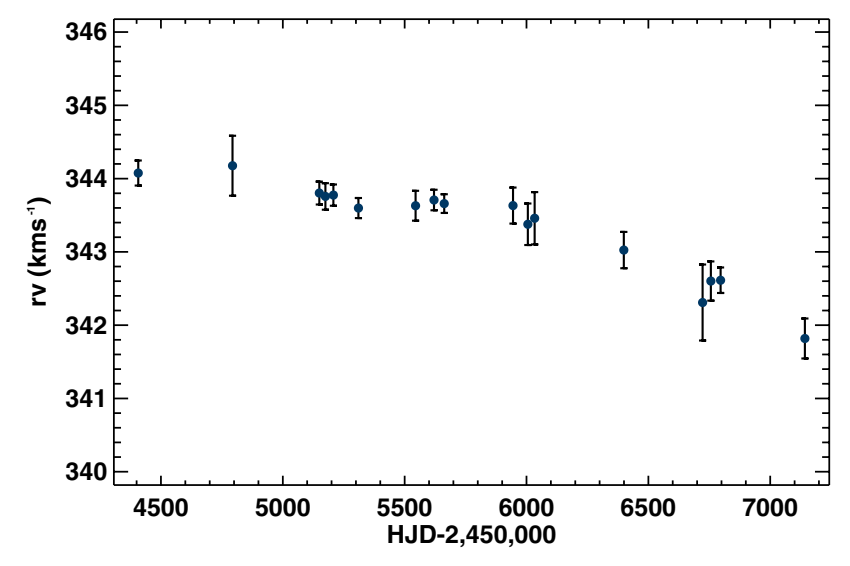

Fig. 2. Radial velocities measured for HE 0959-1424 as function of time, showing a clear decrease over the observing period.

\section{CEMP-rs stars:}

- HE 0017+0055 was monitored extensively in parallel with this programme by Jorissen et al. (2016a), who also discovered its large enhancement of Eu and other $r$-process elements. We have therefore moved it to the CEMP- $r / s$ subgroup of the sample. Moreover, the analysis of our joint data set revealed a low-amplitude, short-period oscillation superimposed on a highly significant long-term trend. For the short-term oscillation, a Keplerian orbit was formally derived from data covering $\approx 8$ full cycles, but the tiny $f(m)$ of $(6 \pm 1) \times 10^{-6} M_{\odot}$ implied an implausibly low orbital inclination of $\approx 2^{\circ}$ (see Sect. 3.2).

An alternative interpretation in terms of stellar pulsations, based on the complete material, was therefore discussed by Jorissen et al. (2016a), who found the oscillations in this star to be similar to those in the CEMP-no star HE 1410+0213 (Paper II). In the end, regardless of the ultimate cause of these short-period, low-level oscillations, the reality of the long-term orbital motion of HE $0017+0055$ is not in doubt, and we retain the star as a binary in our sample.

- HE 0039-2635 (alias CS 29497-034) was observed by both Lucatello et al. (2005) and Barbuy et al. (2005). The former report one measurement from 2002, while the latter reported eleven independent measurements over a span of $\approx 3000$ days between 1995 and 2004 with an rms of $3.4 \mathrm{~km} \mathrm{~s}^{-1}$, and derived an orbital solution with $P=4130$ days and $e=0.2$. We have combined our results with the published data and find a more eccentric orbit with a shorter period $(P=3223$ days, $e=0.46$ ); see Fig. 4 and Table 4 .

- LP 625-44 has published radial-velocity data from the following sources: Norris et al. (1997): five observations during 1988-1996; Aoki et al. (2000): two observations from 1998-2000; and Lucatello et al. (2005): three observations from 2000-2002. None of these authors had sufficient data to compute an orbital solution for this star. However, combining their data with our own extensive series of measurements, and applying offsets between the literature velocities and ours (Norris et al., $-0.14 \mathrm{~m} \mathrm{~s}^{-1}$; Aoki et al., $+451.96 \mathrm{~m} \mathrm{~s}^{-1}$; and Lucatello et al., $+155.87 \mathrm{~m} \mathrm{~s}^{-1}$ ), we could construct a data set covering a total time span of 9582 days. From this, we have computed an orbit with a period of $P=4863$ days and $e=0.35$; see Table 4 and Fig. 4. 
T. T. Hansen et al.: The role of binaries in the enrichment of the early Galactic halo. III.
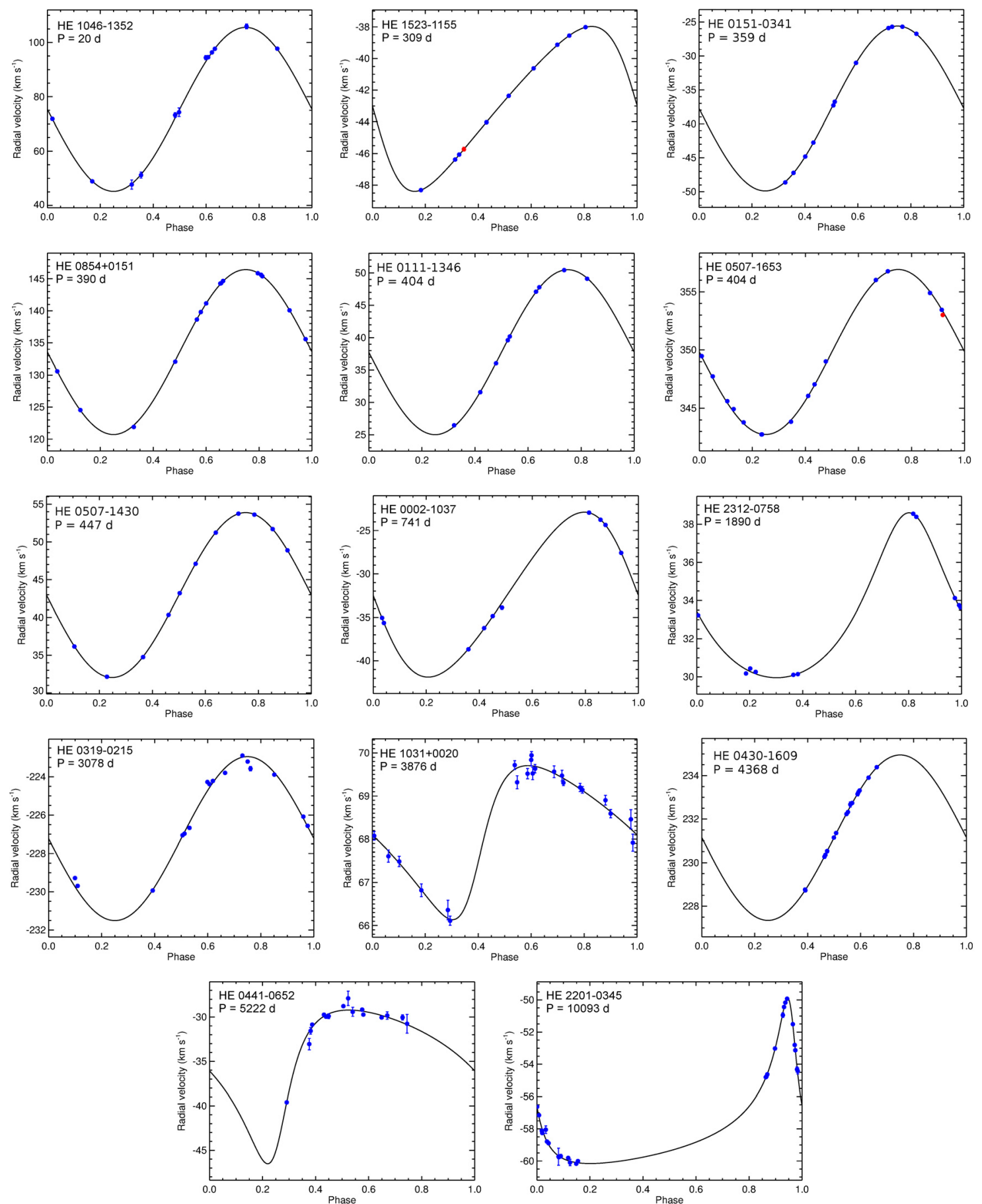

Fig. 3. Orbit solutions for the CEMP-s binaries in our programme. Blue symbols: this work; red symbols: Aoki et al. (2007).

\section{Binary orbital solutions}

Orbital solutions have been obtained for the 17 confirmed binary systems in our sample, except the probable (very) long-period binary HE 0959-1424, which we discussed above. Our final orbital parameters for these 17 systems are listed in Table 4 in order of increasing period, and radial-velocity curves are shown, also in order of increasing period, in Fig. 3 for the CEMP-s stars and in Fig. 4 for the CEMP-r/s stars.

The orbital periods of the binaries among our programme stars range from 20 days to $\approx 30 \mathrm{yr}$, the majority having periods around one year; most of these are in circular orbits. Naturally, for orbits with periods in excess of the $\approx 3000$-day time span of our observations, the orbital parameters have correspondingly larger errors.

Table 4 also lists the Roche-lobe radii of the secondary stars in these systems, calculated by the procedure described in Paper I. For this, we have assumed a mass of $0.8 M_{\odot}$ for the observed metal-poor giant primary stars, and secondary masses of $0.4 M_{\odot}$ (larger if $i=90^{\circ}$ is reached) and $1.4 M_{\odot}$, respectively (minimum and maximum masses for the presumed white dwarf companion). For orbital periods $P \gtrsim 3000$ days, these Rochelobe radii become very uncertain; therefore, only indicative values for $M_{2}=0.6 M_{\odot}$ are given. 
Table 4. Orbital parameters for the binary systems in our sample.

\begin{tabular}{|c|c|c|c|c|c|c|c|c|c|c|c|}
\hline $\begin{array}{l}\text { Parameter } \\
\text { Units }\end{array}$ & $\begin{array}{r}\text { Period } \\
\text { (days) }\end{array}$ & $\begin{array}{r}T_{0} \\
(\mathrm{HJD}) \\
\end{array}$ & $\begin{array}{r}K \\
\left(\mathrm{~km} \mathrm{~s}^{-1}\right) \\
\end{array}$ & $\begin{array}{r}\gamma \\
\left(\mathrm{km} \mathrm{s}^{-1}\right) \\
\end{array}$ & $e$ & $\begin{array}{r}\omega \\
\left({ }^{\circ}\right) \\
\end{array}$ & $\begin{array}{r}a \sin i \\
\left(R_{\odot}\right) \\
\end{array}$ & $\begin{array}{l}f(m) \\
\left(M_{\odot}\right)\end{array}$ & $\begin{array}{r}M_{2} \\
\left(M_{\odot}\right) \\
\end{array}$ & $\begin{array}{r}R_{\text {Roche }} \\
\left(R_{\odot}\right)\end{array}$ & $\begin{array}{r}\sigma \\
\left(\mathrm{km} \mathrm{s}^{-1}\right) \\
\end{array}$ \\
\hline \multicolumn{12}{|c|}{ CEMP- $s$ Stars } \\
\hline \multirow[t]{2}{*}{ HE 1046-1352 } & 20.156 & 51199.88 & 30.19 & +75.37 & 0.00 & 0 & 12.03 & 0.057 & 0.4 & 5.8 & 0.82 \\
\hline & 0.001 & 0.04 & 0.30 & 0.16 & 0.00 & - & 0.01 & 0.008 & 1.4 & 22.0 & \\
\hline \multirow[t]{2}{*}{ HE 1523-1155 } & 309.34 & 57009.3 & 5.21 & -42.94 & 0.272 & 100 & 30.66 & 0.0040 & 0.4 & 31.9 & 0.018 \\
\hline & 0.62 & 2.6 & 0.04 & 0.11 & 0.008 & 2 & 0.18 & 0.0002 & 1.4 & 132.5 & \\
\hline \multirow[t]{2}{*}{ HE $0151-0341$} & 359.07 & 55313.4 & 12.15 & -37.74 & 0.00 & 0 & 86.22 & 0.0667 & 0.45 & 42.2 & 0.08 \\
\hline & 0.21 & 0.7 & 0.03 & 0.03 & 0.00 & - & 0.16 & 0.0004 & 1.4 & 146 & \\
\hline \multirow[t]{2}{*}{ HE $0854+0151$} & 389.85 & 55305.58 & 12.86 & +133.58 & 0.00 & 0 & 99.1 & 0.0859 & 0.5 & 52.0 & 0.13 \\
\hline & 0.07 & 0.07 & 0.03 & 0.02 & 0.00 & - & 0.1 & 0.0003 & 1.5 & 155 & \\
\hline \multirow[t]{2}{*}{ HE 0111-1346 } & 403.81 & 56320.2 & 12.74 & +37.75 & $0.00^{*}$ & 0 & 101.32 & 0.8855 & 0.55 & 56.7 & 0.11 \\
\hline & 0.14 & 0.1 & 0.02 & 0.02 & - & - & 0.08 & 0.0001 & 1.4 & 162 & \\
\hline \multirow[t]{2}{*}{ HE $0507-1653$} & 404.18 & 55840.24 & 7.090 & +349.843 & 0.00 & 0 & 56.63 & 0.0145 & 0.4 & 36 & 0.20 \\
\hline & 0.05 & 0.07 & 0.008 & 0.006 & 0.00 & - & 0.03 & 0.0002 & 1.4 & 156 & \\
\hline \multirow[t]{2}{*}{ HE 0507-1430 } & 446.96 & 55272.71 & 10.927 & +42.961 & 0.0058 & 84 & 96.64 & 0.0604 & 0.44 & 47 & 0.05 \\
\hline & 0.15 & 0.16 & 0.011 & 0.009 & 0.0015 & 11 & 0.06 & 0.0001 & 1.4 & 169 & \\
\hline \multirow{2}{*}{ HE 0002-1037 } & 740.9 & 56622.5 & 9.50 & -32.49 & 0.142 & 85 & 138.0 & 0.064 & 0.4 & 67 & 0.29 \\
\hline & 1.0 & 0.5 & 0.08 & 0.03 & 0.005 & 3 & 0.5 & 0.003 & 1.4 & 242 & \\
\hline \multirow[t]{2}{*}{ HE 2312-0758 } & 1890 & 56536 & 4.33 & +33.16 & $0.26^{*}$ & 0 & 156 & 0.014 & 0.4 & 99 & 0.14 \\
\hline & 57 & 7 & 0.08 & 0.15 & - & - & 6 & 0.005 & 1.4 & 447 & \\
\hline \multirow[t]{2}{*}{ HE 0319-0215 } & 3078 & 53572 & 4.28 & 227.23 & 0.00 & 0 & 260.3 & 0.025 & 0.4 & 142 & 0.34 \\
\hline & 25 & 19 & 0.05 & 0.05 & 0.00 & - & 3.2 & 0.05 & 1.4 & 634 & \\
\hline \multirow[t]{2}{*}{ HE $1031-0020$} & 3867 & 56006 & 1.78 & +68.22 & $0.38^{*}$ & 245 & 126 & 0.002 & 0.6 & 284 & 0.17 \\
\hline & 175 & 54 & 0.07 & 0.06 & - & - & 28 & 0.035 & & & \\
\hline \multirow[t]{2}{*}{ HE 0430-1609 } & 4368 & 58873 & 3.80 & +231.15 & $0.0^{*}$ & 0 & 328 & 0.025 & 0.6 & 310 & 0.04 \\
\hline & 198 & 96 & 0.15 & 0.06 & - & - & 20 & 0.002 & & & \\
\hline \multirow[t]{2}{*}{ HE 0441-0652 } & 5223 & 58408 & 8.7 & -34.58 & $0.48^{*}$ & 217 & 785 & 0.24 & 0.72 & 540 & 0.64 \\
\hline & 628 & 308 & 3.3 & 1.95 & - & - & 193 & 0.26 & & & \\
\hline \multirow{2}{*}{ HE 2201-0345 } & 10093 & 55696 & 5.13 & -58.08 & 0.67 & 28 & 749 & 0.055 & 0.6 & 556 & 0.17 \\
\hline & 1656 & 8 & 0.05 & 0.24 & 0.04 & 1 & 129 & 0.029 & & & \\
\hline \multicolumn{12}{|c|}{ CEMP- $r / s$ Stars } \\
\hline \multirow[t]{2}{*}{ HE 0039-2635 } & 3223 & 51900 & 6.9 & -46.5 & $0.46^{*}$ & 239 & 424 & 0.09 & 0.6 & 264 & 1.32 \\
\hline & 36 & 12 & 0.5 & 10.4 & - & - & 139 & 0.53 & & & \\
\hline \multirow[t]{2}{*}{ HE $0017+0055$} & 3529 & 55407 & 1.57 & -80.39 & 0.43 & 312 & 99 & 0.001 & 0.6 & 64 & 0.33 \\
\hline & 236 & 40 & 0.06 & 0.05 & 0.05 & 8 & 9 & 0.035 & & & \\
\hline \multirow[t]{2}{*}{ LP 625-44 } & 4863 & 56007 & 6.35 & +33.63 & $0.35^{*}$ & 245 & 571 & 0.10 & 0.6 & 332 & 0.42 \\
\hline & 12 & 16 & 0.04 & 0.06 & - & - & 18 & 0.01 & & & \\
\hline
\end{tabular}

Notes. Mean errors are given below each parameter. ${ }^{(*)}$ Eccentricity fixed in the solution.

\subsection{Binary fraction and distribution of orbital eccentricities}

With 17 confirmed and one likely binary systems, the binary frequency of our sample is $77-82 \pm 10 \%$, taking Poisson sampling errors into account, but $\approx 20 \%$ of the stars appear to be single. Thus, the frequency of spectroscopic binaries among CEMP- $s$ stars is clearly much higher than among both Population I and II giants in the field (Mermilliod et al. 2007; Carney et al. 2003), but as discussed in Sect. 3.2, it is probably not quite the $100 \%$ surmised by Lucatello et al. (2005). That $\approx 20 \%$ of the stars remain single suggests that, like the CEMP-no stars of Paper II, they did not receive their high carbon and $s$-process-element abundances via mass transfer from a former AGB companion; another scenario must be invoked (see Sect. 7).

Figure 5 shows our binary systems (Table 4) in the periodeccentricity diagram (red plus signs and red and blue triangles), along with comparison samples of 141 giant binary members of open clusters of all ages by Mermilliod et al. (2007) and Mathieu et al. (1990; black dots), and 16 metal-poor binaries from Carney et al. (2003; black crosses). HE 0959-1424 has been included in the plot with a fictitious period of 15000 days to indicate that its period is likely very long, but presently unknown. The three CEMP-r/s stars (HE 0017+0055, HE 0039-2635, and LP 625-44; blue triangles) have similar eccentric orbits with long periods (of order a decade or more), and are among the longest-period stars in our sample (Table 4). However, the present sample is too small to claim any difference in binary characteristics between CEMP- $r / s$ and CEMP- $s$ stars (see further discussion in Sect. 6).

The period-eccentricity diagram shown in Fig. 5 exhibits the expected overall feature (Jorissen et al. 1998, 2016b) of (near-) circular orbits up to a cut-off that depends on the age of the systems, but can be estimated at $\approx 200$ days for Population I and Population II giants of all ages. This is commonly ascribed to tidal circularisation of the orbits, which is more advanced the older the star; the few moderately eccentric, shorter-period binaries are probably younger and/or more metal-poor stars in uncertain stages of evolution.

The CEMP- $s$ and CEMP- $r / s$ binaries shown in Fig. 5 indicate a cut-off period in the range 500-700 days, suggestive of larger (AGB) secondaries. Two systems, HE 1046-1352 and HE 1523-1155, are exceptions to this general trend. HE 1046-1352, with a period of only 20 days, does have the expected circular orbit, but the calculated Roche-lobe radii for an average-mass white dwarf secondary are too small to accommodate even a normal giant star, let alone an AGB star 
T. T. Hansen et al.: The role of binaries in the enrichment of the early Galactic halo. III.
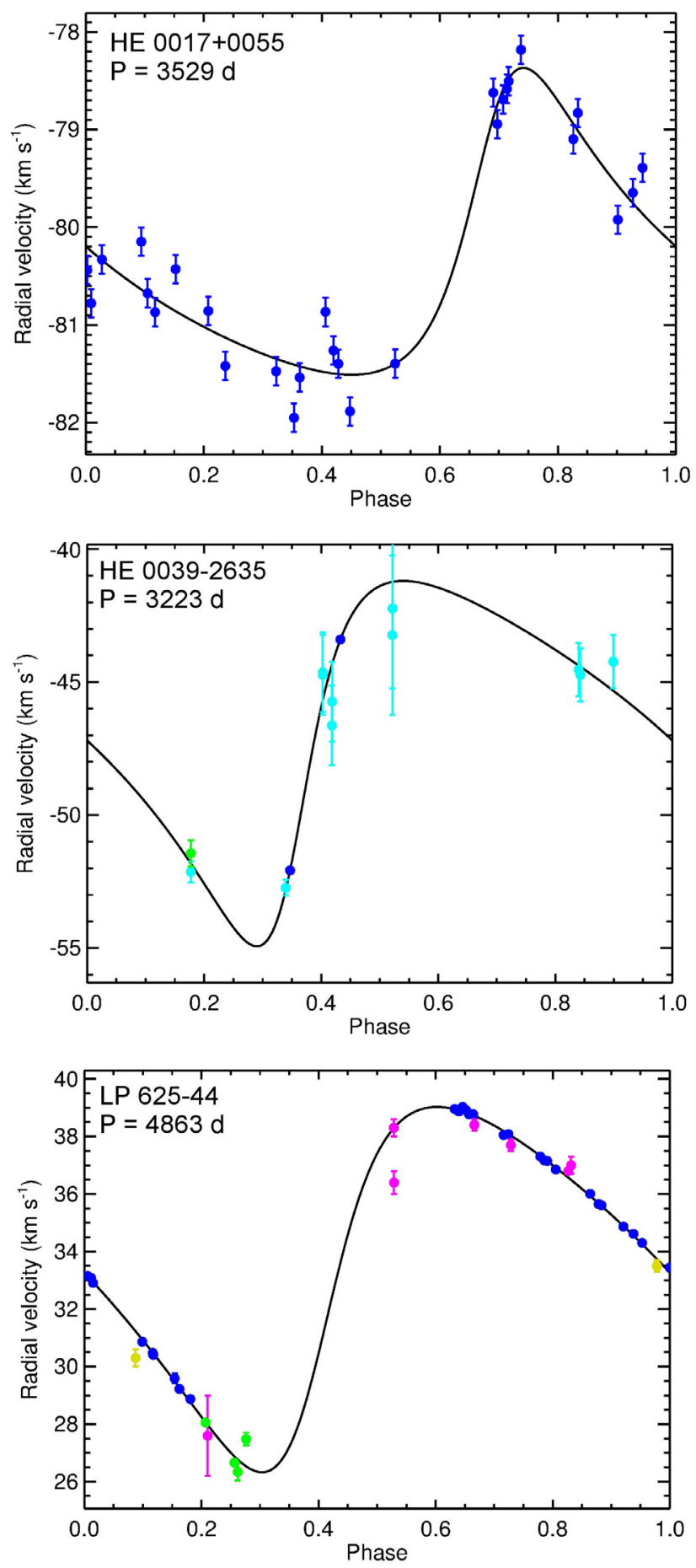

Fig. 4. Orbits for the CEMP-r/s stars. Symbols: Blue: this work; magenta: Norris et al. (1997); green: Lucatello et al. (2005); yellow: Aoki et al. (2000); light blue: Barbuy et al. (2005).

of $\approx 200 R_{\odot}$. HE $1046-1352$ could be a misclassified dwarf or the result of a common-envelope phase of evolution, similar to the even shorter-period system HE 0024-2523 discussed by Lucatello et al. (2003) on the basis of its high spectroscopic $\log g$, remarkable abundance pattern, and high rotation.

HE 1523-1155 deviates from expectation in the opposite sense in that it has a highly significant eccentricity of $e=0.3$, yet has a shorter period ( 309 days) than the $\approx 600$-day cut-off defined by the several systems with circular orbits and periods near 500 days. As noted above, the period is observationally strongly constrained, so any additional observations could

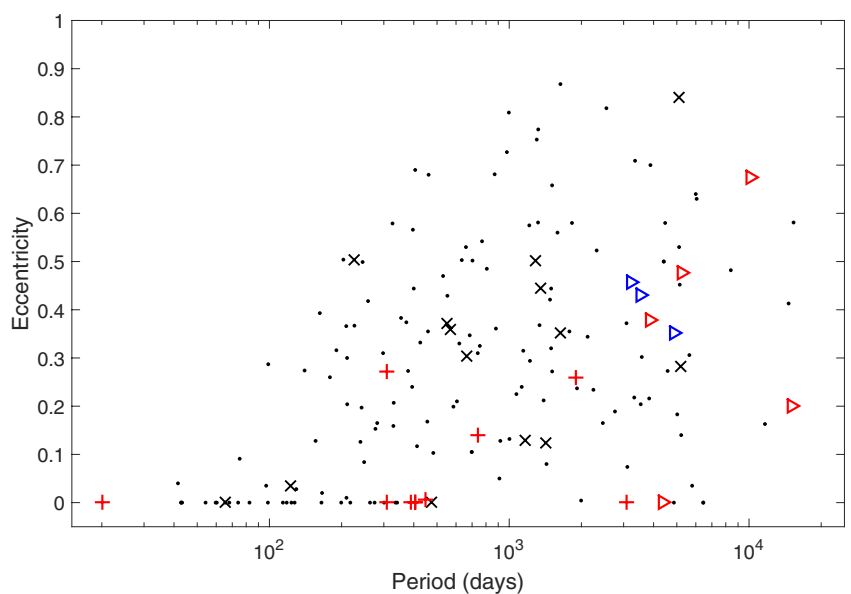

Fig. 5. Period-eccentricity diagram for the binary systems in our sample. Red and blue symbols denote CEMP- $s$ and CEMP- $r / s$ stars, respectively. Systems with periods $P<3000$ days are plotted as right-pointing triangles to indicate that the orbital parameters are still uncertain; their periods, in particular, may well be even longer. Black dots and crosses: Comparison sample of 141 giant binary members of Galactic open clusters of all ages (Mermilliod et al. 2007; Mathieu et al. 1990) and 16 metal-poor and C-normal binaries from Carney et al. (2003).

only confirm the currently derived eccentricity or lead to an even higher value. One might suspect whether HE 1523-1155 could also be a dwarf. However, the spectroscopic $\log g=1.6$ derived by Aoki et al. (2007) from a Subaru spectrum with $R \approx 50000$ indicates a giant classification for this star.

\section{Formation of CEMP-s stars via mass transfer}

In analogy with the higher metallicity $\mathrm{CH}$ and $\mathrm{Ba}$ stars, and based on recent detailed models for them (e.g., Abate et al. 2015a and references therein), CEMP- $s$ stars can be produced through local mass transfer from an AGB binary companion. However, challenges for this scenario remain, such as: (i) can models account for their observed frequencies at low metallicity? Previous attempts fall well short of matching the observations, but progress is being made; see Abate et al. 2015b; (ii): can the models reproduce the observed distribution of periods and (final) separations of the binary components?; and (iii): can the models account for the detailed abundance patterns of CEMP$s$ (and CEMP- $r / s$ ) stars? Final resolution of these questions will require substantially larger samples of CEMP- $s$ (and CEMP- $r / s$ ) stars with precise long-term radial-velocity monitoring and high $\mathrm{S} / \mathrm{N}$. high-resolution spectra. Below we speculate on what can be inferred from the present sample.

\subsection{Mass-transfer mechanisms}

The transfer of mass from an AGB companion star onto the lowmass star we observe today as a CEMP- $s$ star can happen in three more-or-less efficient ways: Roche-lobe overflow (RLOF), wind transfer, or wind-assisted Roche-lobe overflow (WRLOF).

Roche-lobe overflow occurs when a binary component expands beyond its Roche-lobe radius. Mass can then be transferred to the companion through the inner Lagrangian point (L1). This is the most efficient way to transfer mass in a binary system, but for RLOF to operate, the separation of the two stars in the system must be relatively small; if the stars are too far apart, neither of them ever fills its Roche lobe. On the other hand, if the stars are too close, they enter a common-envelope phase. If 
a binary system undergoes RLOF, this circularises the orbit very effectively; even more so if it enters a common-envelope phase. The RLOF from stars with large convective envelopes, such as AGB stars, is generally believed to be unstable and often develops into a common-envelope phase (Kopal 1959; Paczyński 1965, 1976). Mass transfer during the common envelope phase is very inefficient and usually considered to be negligible (Ricker \& Taam 2008); therefore, RLOF is generally not considered a possible formation mechanism for CEMP-s stars.

From Table 4, it appears that the calculated secondary Roche-lobe radii for our binary systems are only large enough to accommodate an AGB star of $\approx 200 R_{\odot}$ in systems with orbital periods $P \gtrsim 1000$ days and massive present-day WD companions. This, along with the obstacles of common-envelope evolution as mentioned above, would seem to rule out the direct RLOF of mass transfer in shorter-period binaries and favour the WRLOF mode discussed below.

Another method for mass transfer in a binary system is wind transfer, where the low-mass star is exposed to the wind of the AGB star, and can thereby accrete mass (Bondi \& Hoyle 1944). This Bondi-Hoyle-Lyttleton (BHL) model of wind mass transfer assumes that the wind of the AGB star does not interact with the orbit of the accreting star, which requires the wind velocity $\left(v_{\text {wind }}\right)$ to be much higher than the orbital velocity $\left(v_{\text {orbit }}\right)$; thus, this type of mass transfer is an option only if $v_{\text {wind }} \gg v_{\text {orbit }}$.

This is not always the case for wide binary systems, however. If $P \approx 10^{4}$ days, the orbital velocity is $\approx 10 \mathrm{~km} \mathrm{~s}^{-1}$, and the wind from AGB stars can have velocities of $5-30 \mathrm{~km} \mathrm{~s}^{-1}$ (Vassiliadis $\&$ Wood 1993). Boffin \& Jorissen (1988) explored the possibility of creating Ba stars (the higher metallicity equivalent of CEMP- $s$ stars) via wind transfer in detached binary systems using the BHL wind-accretion scenario, and they concluded that the $\mathrm{Ba}$ stars could indeed have been formed in this way. This type of mass transfer allows orbital periods to remain long and orbits to not be circularised.

A third option is the WRLOF mass-transfer mechanism, which can occur in systems where the wind of the donor star is gravitationally confined to the Roche-lobe of the accreting star. The wind can then be focussed towards the orbital plane of the binary system and transferred to the secondary through the L1 point. This type of transfer can be significantly more efficient than BHL wind transfer (Mohamed \& Podsiadlowsky 2007). The WRLOF mechanism facilitates mass transfer in binary systems that are too wide for mass transfer via conventional RLOF. The majority of the material that is not accreted by the secondary is lost through the outer Lagrangian points (L2 and L3), and thus carries away angular momentum from the system, thereby shrinking the orbit (Abate et al. 2013).

Abate et al. (2015a) attempted to reproduce the chemical abundance pattern and orbital properties of 15 known CEMP- $s$ binary systems. To do this, they compared two models: (1) a WRLOF model with angular momentum loss calculated assuming a spherical symmetric wind; and (2) an enhanced BHL windtransfer model with efficient angular momentum loss.

Depending on the specific model adopted for the angularmomentum loss, the binary system can widen or shrink in response. Models with WRLOF and a spherically symmetric wind widen the orbit as mass is transferred, i.e., small initial periods and consequently small secondary masses are required. Otherwise, they would overflow their Roche lobes, which would lead to a common-envelope phase with no accretion onto the companion. Because the WRLOF is not very efficient for small separations, only small amounts of material is transferred, and this model fails to match the measured abundances of the CEMP- $s$ stars.

In contrast, the BHL wind-transfer model with enhanced angular-momentum loss predicts high accretion efficiency (also for systems with relatively short periods), and the enhanced angular-momentum loss ensures that the systems always shrink in response to the mass loss. Thus, even systems that are initially very wide can end up being relatively close when the primary has become a white dwarf.

The majority of their CEMP-s binary systems were best reproduced by the second model, which the authors doubted is realistic, but which emphasised the need for very efficient mass transfer to create the abundance patterns observed in the CEMP- $s$ stars. The systems modelled by Abate et al. (2015a) have periods ranging between 3.33 and 4280 days, and they find initial periods of 1170 to 130000 days for these systems.

Hence, Abate et al. (2015a) conclude that, to reproduce the observations, “... it is generally necessary that the modelled binary systems lose efficiently angular momentum and transfer mass with high accretion efficiency". In some systems, a common-envelope phase is even needed to shrink the orbits to their current size. This is particularly true for the binary system HE 0024-2523, with a period of only 3.41 days (Lucatello et al. 2003).

\subsection{Nucleosynthesis challenges}

Aside from the challenges of efficiently transferring mass in a binary system, most AGB models also face difficulty in reproducing the detailed elemental-abundance patterns found in CEMP- $s$ stars. For instance, Bisterzo et al. (2012) fitted the abundance patterns of 94 individual CEMP- $s$ and CEMP- $r / s$ stars with yields from AGB-star models. The outcome of that exercise demonstrated that the models have problems reproducing the $\mathrm{C}$ and $\mathrm{N}$ abundances, as well as the ${ }^{12} \mathrm{C} /{ }^{13} \mathrm{C}$ ratios reported for CEMP stars. Carbon is generally over-produced in their models. Combined with the observed low ${ }^{12} \mathrm{C} /{ }^{13} \mathrm{C}$ ratios (see e.g. Ryan et al. 2005 and Hansen et al. 2015a, and references therein), this points towards a large amount of mixing that is not included in their models. Recent work by Abate et al. (2015a,b), employing models of AGB nucleosynthesis production from Karakas (2010), reaches better agreement for some stars, while for others they have the same problem as Bisterzo et al. (2012).

In addition, predictions of the $s$-process-element distributions present difficulties. The AGB models of Bisterzo et al. (2012) predict roughly similar abundances (within 0.3 dex) of the first-peak (Sr, Y, Zr) and second-peak elements (Ba, La, Ce, $\mathrm{Pr}$, and Nd), while the derived abundances for CEMP- $s$ stars exhibit an internal spread in these elements of more than 0.5 dex. Barium is often found to be more enhanced than the other second-peak elements.

Placco et al. (2013) attempted to match the measured abundances of CEMP- $s$ stars (without scaling the pattern to the Ba abundance) with yields from the AGB models of Karakas (2010), taking the dilution across the binary system into account. In this case, the elements from the first $s$-process peak were under-produced, while $\mathrm{Ba}$ and $\mathrm{Pb}$ were over-produced by the model. Alternatively, Hansen et al. (2015a) compared yields from the F.R.U.I.T.Y database of Cristallo et al. (2009, 2011) to the measured abundance pattern of two CEMP- $s$ stars. These models failed to reproduce the large amounts of $\mathrm{C}$ and $\mathrm{N}$ detected in the stars, and also have problems matching the abundances derived for the light neutron-capture elements ( $\mathrm{Sr}, \mathrm{Y}$, and $\mathrm{Zr}$ ) of the stars. 
Near-ultraviolet (NUV) observations of CEMP- $s$ stars also help constrain the theoretical models. Placco et al. (2015) studied two bright CEMP- $s$ stars with HST/STIS spectra, which allowed them to determine abundances for elements such as Ge, $\mathrm{Nb}, \mathrm{Mo}, \mathrm{Lu}, \mathrm{Pt}$, and Au. They also matched the abundance pattern with the latest models from Abate et al. (2015a), which take into account not only the AGB evolution, but also the dynamics of the binary system. Even though NUV abundance determinations for CEMP stars are challenging to obtain, they are important inputs for such model-matching exercises.

\subsection{Dilution of the accreted material}

The accreted material also mixes with interior material from the accreting stars via thermohaline mixing (Stancliffe et al. 2007), which results in the dilution of the accreted material and a change in its abundance pattern. Attempts to model this effect have been made, but the degree to which the transferred material is diluted on the surface of the receiving star is still very poorly constrained (Stancliffe et al. 2007; Stancliffe \& Glebbeek 2008).

The accreted material is also mixed with the material of the accreting star during any dredge-up episodes that occur as the low-mass, CEMP-s star evolves past the main-sequence stage. This is the case for some of the stars modelled by Abate et al. (2015a), which need to have even more mass transferred to account for the mixing occurring during first dredge-up in order to match the abundance pattern of the observed CEMP- $s$ star. In addition, Richard et al. (2002) argue that the degree of dilution depends on the chemical composition of the accreted material. These different mixing effects change the composition of the surface of the CEMP- $s$ star, and introduce yet another source of uncertainty into the mass-transfer models.

\section{The CEMP-r/s stars}

Europium has been detected in four stars of our sample (see Table 2), indicating an enhancement in $r$-process as well as $s$-process elements. One of these, CS 30301-015, we have now classified as a CEMP- $s$ star; it is also a single star. The other three, HE 0017+0055, HE 0039-2635, and LP 625-44, are classified as CEMP- $r / s$ stars and are long-period binaries with orbital properties that are indistinguishable from those of CEMP- $s$ stars.

Based on Ba and Eu abundances for CEMP- $s$ and CEMP- $r / s$ stars, Lugaro et al. (2012) argued that the abundance patterns found for the stars cannot come from the same single AGB star source, which is essentially the opposite of the claim by Allen et al. (2012) that the astrophysical origin of the CEMP- $s$ and CEMP- $r / s$ stars is one and the same.

It has also been speculated that the CEMP- $r / s$ stars may exhibit the chemical signature of a different origin, i.e., the intermediate neutron-capture $(i$-)process. The $i$-process, operating at neutron fluxes between the $s$ - and $r$-process, was first proposed by Cowan \& Rose (1977) to occur in evolved red giants. This was supported by Masseron et al. (2010), who argued that CEMP- $r / s$ stars could be the result of mass transfer from more massive AGB stars, where the neutron source ${ }^{22} \mathrm{Ne}(\alpha, \mathrm{n})^{25} \mathrm{Mg}$ is active during the thermal pulses. This source is predicted to produce sufficiently high neutron exposures to not only synthesise elements traditionally associated with the $s$-process, but also elements such as Eu, which are normally associated with the $r$-process (Goriely \& Siess 2005). More recently, Bertolli et al. (2013) also found that the abundance pattern of CEMP-r/s stars could be matched with the yields from the $i$-process, which are thought to occur in high-mass "super-AGB" stars.
Table 5. Orbital elements for CEMP- $r / s$ binary stars from the literature.

\begin{tabular}{lrrl}
\hline \hline Stellar ID & Period & $e$ & Ref. \\
\hline CS 22948-027 & 427 & 0.02 & Barbuy et al. (2005) \\
CS 29497-030 & 346 & 0.30 & Preston \& Sneden (2000) \\
HD 224959 & 1273 & 0.18 & McClure \& Woodsworth (1990) \\
\hline
\end{tabular}

We have searched the literature for CEMP stars with $r$ - and $s$-process element abundances such that they would qualify as CEMP-r/s stars, and with published radial-velocity data. Nine such stars show radial-velocity variations, but only three have published orbital elements (see Table 5); five show no variation. However, the information on their stage of evolution (essentially a spectroscopic $\log g$ ) is ambiguous.

Accordingly, no significant new information can be derived from a $P$-e diagram such as that in Fig. 5, except that the orbital periods of the certified giant CEMP-r/s stars are long and their eccentricities are significant, but moderate, which is very similar to those of the rest of the CEMP- $r / s$ stars. Larger samples of CEMP- $r / s$ stars with detailed information on binary orbital parameters and a more complete inventory of heavy neutroncapture elements are clearly required for further progress.

\section{Composition of the single stars}

Four stars in our sample exhibit constant velocities over our observing period, and are judged to be single (see Fig. 1 and Sect. 3.2): HE 0206-1916, HE 1045+0226, HE 2330-0555, and CS 30301-015. The atmospheric parameters for these single stars are listed in Table 6. They are all giants, which may have experienced a first dredge-up (FDU) episode. During this FDU, $\mathrm{H}$, He, and products of the $\mathrm{CN}$ cycle are mixed to the surface, resulting in a small decrease in $[\mathrm{C} / \mathrm{Fe}]$ along with an increase in $[\mathrm{N} / \mathrm{Fe}]$ at the surface of the star (the precise abundance changes depend on the mass and metallicity of the star and the extent of the FDU; see e.g. Karakas \& Lattanzio 2014). Thus, no great change of their surface abundances is expected to have occurred, and a different channel must be invoked to account for the origin of the carbon and $s$-process-element enhancements found in these stars.

To explore this possibility, we have plotted the abundance information we have in common for all of the stars, namely the $\mathrm{C}, \mathrm{Fe}$, and $\mathrm{Ba}$ abundances; see Figs. 6 and 7. The single stars (red dots) exhibit no clear difference in abundance signatures for these elements as compared with the binary stars (blue dots and stars). More detailed abundance analyses, spanning a greater range of elements than is available at present, may reveal differences in the abundance patterns of the single stars vs. those found in binary systems, which could help constrain the likely formation scenario of these stars.

\subsection{Potential sources of carbon and s-process elements in the early Universe}

The carbon excess detected in the single CEMP- $s$ stars may have a similar origin as the carbon excess seen in the CEMP-no stars, which is believed to be due to either winds from the so-called "spinstars", massive, rapidly-rotating, metal-free stars (Meynet et al. 2006; Hirschi 2007; Maeder et al. 2015), or to faint supernovae with mixing and fallback as suggested by Umeda \& Nomoto (2003).

Spinstars are also potentially a main contributor to $s$-processelement abundances in the early Universe. It was first suggested by Pignatari et al. (2008) that rotation-induced mixing in these stars enhances the production of $s$-process elements via the weak 
Table 6. Stellar parameters for single stars.

\begin{tabular}{lcccl}
\hline \hline Stellar ID & $T_{\text {eff }}(\mathrm{K})$ & $\log g$ & {$[\mathrm{Fe} / \mathrm{H}]$} & Ref. \\
\hline HE 0206-1916 & 5200 & 2.7 & -2.09 & Aoki et al. (2007) \\
HE 1045+0226 & 5077 & 2.2 & -2.20 & Cohen et al. (2013) \\
HE 2330-0555 & 4900 & 1.7 & -2.78 & Aoki et al. (2007) \\
CS 30301-015 & 4750 & 0.8 & -2.64 & Aoki et al. (2002a) \\
\hline
\end{tabular}

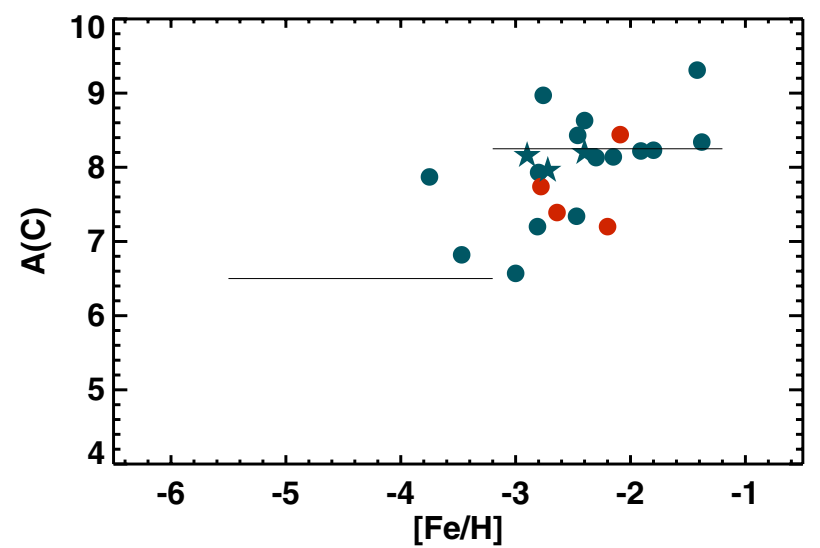

Fig. 6. Absolute $\mathrm{C}$ abundances for our programme stars. Dots represent CEMP- $s$ stars, star symbols CEMP- $r / s$ stars; red symbols show single stars, blue symbols certified binaries. The two C-bands first noted by Spite et al. (2013) are indicated with horizontal lines.

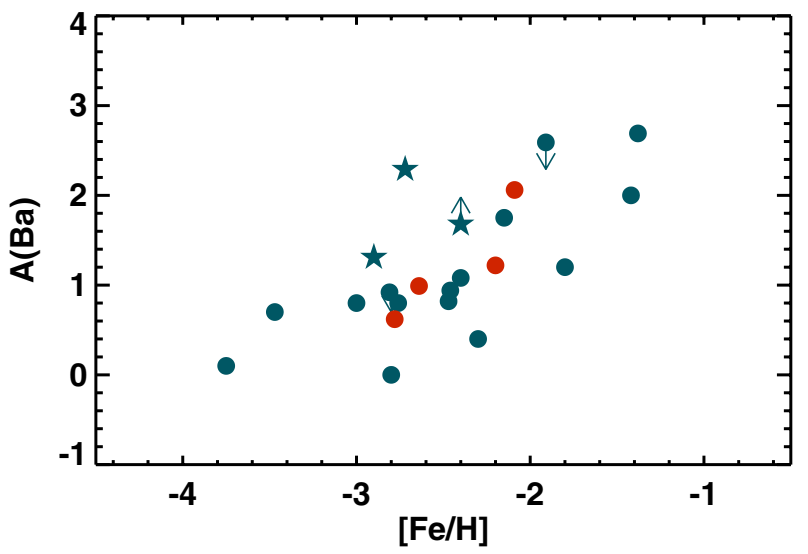

Fig. 7. Absolute Ba abundances for our programme stars. Dots represent CEMP- $s$ stars, star symbols indicate CEMP- $r / s$ stars; red symbols show single stars, blue symbols indicate definite binaries.

$s$-process. Later, Frischknecht et al. (2012, 2016) explored the yields of the weak $s$-process in spinstars. They found that mainly light $s$-process elements $(\mathrm{Sr}, \mathrm{Y}, \mathrm{Zr}$ ) are produced in this environment, usually with $[\mathrm{Sr} / \mathrm{Ba}]>0$; only in extreme cases does the production run all the way through to $\mathrm{Pb}$.

Abundances of $\mathrm{Sr}, \mathrm{Ba}$, and $\mathrm{Pb}$ have only been reported in the literature for one of our single stars, CS 30301-015; with $[\mathrm{Sr} / \mathrm{Ba}]=-1.15$ and $[\mathrm{Pb} / \mathrm{Fe}]=+1.7$ (Aoki et al. 2002a), it does not match the predictions from Frischknecht et al. (2012). On the other hand, detailed models of the abundance patterns generated by spinstars have not yet matured to the point where meaningful star-by-star comparisons can be carried out. Such models might help to identify the progenitors of the single CEMP- $s$ stars.

\subsection{Carbon bands}

Spite et al. (2013) first suggested the existence of two bands in the absolute carbon abundances of CEMP stars: a high-C band at $A(\mathrm{C}) \approx 8.25$ and a low-C band at $A(\mathrm{C}) \approx 6.5$. The existence of these two bands for CEMP stars has been confirmed with larger samples by Bonifacio et al. (2015) and Hansen et al. (2015a). The high-C band is primarily populated by relatively more metal-rich stars $([\mathrm{Fe} / \mathrm{H}] \gtrsim-3)$ of the CEMP-s subclass, while the low-C band is primarily populated by more metal-poor stars of the CEMP-no subclass. While this general behaviour is supported by the CEMP-no (Fig. 4 of Paper II), CEMP- $s$, and CEMP-r/s (Fig. 6) stars in our programme, there are clear exceptions. For instance, in Fig. 6 a substantial number of stars lie in the transition zone between the two carbon bands, and two stars, classified as CEMP- $s$ stars on the basis of their $[\mathrm{Ba} / \mathrm{Fe}]$ abundance ratios, are nevertheless found close to the low-C band, which is thought to be predominantly associated with CEMP-no stars.

It is remarkable that these stars, HE 0430-1609 and HE 2312-0758, are two of the three stars with the lowest metallicity in our sample. Both stars are confirmed binaries with longperiod orbits. The presence of these stars on the low C-band could be due to less-efficient mass transfer in these systems. Perhaps no mass-transfer event has occurred in these systems at all, and the CEMP- $s$ stars received their $\mathrm{C}$ and $s$-processelement excesses from sources similar to the single CEMP- $s$ stars. The lowest metallicity CEMP- $s$ star in our programme, HE 0002-1037, lies close to the high-C band, similar to the star HE 0959-1424, which is at the high-metallicity limit for stars in our programme and exhibits a carbon abundance that is well above the high- $\mathrm{C}$ band.

The single stars in our sample form a heterogeneous group. Three of the stars, HE 1045-0226, CS 30301-015, and HE 2330-0555, lie in the transition zone between the carbon bands, while the fourth, HE 0206-1916, lies on the high-C band.

There clearly remains much work in order to assess the meaning, and possible astrophysical significance, of the suggested carbon bands. Whether this turns out to be a primary behaviour with deviations that occur for individual stars depending on their precise nucleosynthesis histories, or it is correlated with some other factor related to the production and/or dilution of the carbon due to mixing, this work awaits larger samples with available radial-velocity monitoring and high-S/N spectroscopy.

\section{Conclusions}

Our systematic and precise long-term radial-velocity monitoring programme of 22 CEMP- $s$ and CEMP- $r / s$ stars has led to a high frequency, $82 \pm 10 \%$, of detected binaries in the sample, confirming that the characteristic abundance pattern of the CEMP- $s$ stars is coupled with the binary nature of most of these stars. Indeed, it has been taken for granted up to now that all CEMP- $s$ stars originated from mass transfer in a binary system, a basic assumption that underpins all previous theoretical modelling of these stars.

Four of our programme stars $(18 \%)$ do, however, appear to be truly single and contradict this basic assumption, as do in fact the entire group of CEMP-no stars discussed in Paper II. This underscores the need for an efficient, distant source of $s$-process elements in the early Universe, whether that source might be spinstars or another class of progenitor. Future detailed abundance analyses of these single stars may reveal signatures in their abundance patterns that will help to constrain the nature of the early $\mathrm{C}$ and $s$-process-element production sites and the processes by which these fresh elements were implanted in the natal clouds of the observed CEMP- $s$ stars.

For the binary CEMP- $s$ stars, we highlight a number of respects, notably the detailed treatment of the physics of the mass 
T. T. Hansen et al.: The role of binaries in the enrichment of the early Galactic halo. III.

transfer process, in which current models still fail to reproduce their observed orbital parameters and elemental abundance patterns.

The three CEMP-r/s stars in our sample are all long-period binaries, hinting that the origin of these stars is similar to that of the CEMP- $s$ stars themselves. However, the available data are much too sparse to be conclusive on such key points as whether their excesses of $\mathrm{C}$ and neutron-capture elements stem from the same nuclear source(s), and whether the latter are due to a special form of the $s$-process or to the $i$-process, which has been speculated to operate in the so-called "super-AGB" stars.

Larger samples of binary CEMP- $s$ and CEMP- $r / s$ stars with well-determined orbits, evolutionary status, and detailed abundance patterns are required for further refinement of the theoretical models for the origin of these stars. The accurate trigonometric parallaxes and accompanying precise multi-epoch photometry for many CEMP stars in the field to be expected from the ESA space mission Gaia will soon provide further crucial information on the stage of evolution and pulsational properties of these stars.

Acknowledgements. This paper is based on observations made with the Nordic Optical Telescope, operated by the Nordic Optical Telescope Scientific Association at the Observatorio del Roque de los Muchachos, La Palma, Spain, of the Instituto de Astrofísica de Canarias. We thank numerous NOT staff members and students for readily and very efficiently obtaining most of the many observations for us in service mode. We also thank Carlo Abate for clarifying the response of his modelled binary systems to the different modes of angularmomentum loss. Finally, we thank the anonymous referee for a very careful and incisive report that led to substantive clarifications, revisions, and improvements in the paper. This work was supported by Sonderforschungsbereich SFB 881 "The Milky Way System" (subproject A4) of the German Research Foundation (DFG). J.A. and B.N. gratefully acknowledge financial support from the Danish Natural Science Research Council and the Carlsberg Foundation. T.C.B., V.M.P., and J.Y. acknowledge partial support for this work from grants PHY 08-22648; Physics Frontier Center/Joint Institute or Nuclear Astrophysics (JINA), and PHY 14-30152; Physics Frontier Center/JINA Center for the Evolution of the Elements (JINA-CEE), awarded by the US National Science Foundation.

Note added in proof. After this paper was accepted, we were able to obtain a final FIES spectrum of HE 0430-1609. Remarkably, this velocity still fits two very different orbits with the same rms error of only $40 \mathrm{~m} \mathrm{~s}^{-1}$. We retain the longer-period circular orbit as the most likely and have updated the tables and figures accordingly.

\section{References}

Abate, C., Pols, O. R., Izzard, R. G., Mohamed, S. S., \& de Mink, S. E. 2013, A\&A, 552, A26

Abate, C., Pols, O. R., Karakas, A., \& Izzard, R. G. 2015a, A\&A, 576, A118

Abate, C., Pols, O. R., Stancliffe, R. J., et al. 2015b, A\&A, 581, A62

Allen, D. M., Ryan, S. G., Rossi,S., Beers, T. C., \& Tsangarides, S. A. 2012, A\&A, 548, A34

Aoki, W., Norris, J. E., Ryan, S. G., Beers, T. C., \& Ando, H., 2000, ApJ, L97

Aoki, W., Ryan, S. G., Norris, J. E., et al. 2002a, ApJ, 580, 1149

Aoki, W., Ando, H., Honda, S., et al. 2002b, PASJ, 54, 427

Aoki, W., Beers, T. C., Norris, J. E., Ryan, S. G., \& Tsangarides, S. A. 2007, ApJ, 655, 492

Barbuy, B., Spite, M., Spite, F., et al. 2005, A\&A, 429, 1031

Beers, T. C., \& Christlieb, N. 2005, ARA\&A, 43, 531

Beers, T. C., Preston, G. W., \& Shectman, S. A. 1985, AJ, 90, 2089

Beers, T. C., Preston, G. W., \& Shectman, S. A. 1992, AJ, 103, 1987

Beers, T. C., Flynn, C., Rossi, S., et al. 2007a, ApJS, 168, 128

Beers, T. C., Sivarani, T., Marsteller, B., et al. 2007b, AJ, 133, 1193

Beers, T. C., Norris, J. E., Placco, V. M., et al. 2014, ApJ, 794, 58

Bertolli, M. G., Herwig, F., Pignatari, M., \& Kawano, T. 2013, arXiv e-prints [arXiv: 1310.4578$]$

Bisterzo, S., Gallino, R., Straniero, O., Cristallo, S., \& Käppeler, F. 2012, MNRAS, 422, 849
Boffin, H. M. J., \& Jorissen, A. 1988, A\&A, 205, 155

Bondi, H., \& Hoyle, F. 1944, MNRAS, 104, 273

Bonifacio, P., Caffau, E., Spite, M., et al. 2015, A\&A, 579, A28

Carney, B. W., Latham, D. W., Stefanik, R. P., et al. 2003, AJ, 125, 293

Christlieb, N., Schörck, T., Frebel, A., et al. 2008, A\&A, 484, 721

Cohen, J. G., McWilliam, A., Shectman, S., et al. 2006, AJ, 132, 137

Cohen, J. G., Christlieb, N., Thompson, I., et al. 2013, ApJ, 778, 56

Cowan, J. J., \& Rose, W. K. 1977, ApJ, 212, 149

Cristallo, S., Straniero, O., Gallino, R., et al. 2009, ApJ, 696, 797

Cristallo, S., Piersanti, L., Straniero, O., et al. 2011, ApJS, 197, 17

Duquennoy, A., \& Mayor, M. 1991, A\&A, 248, 485

Frebel, A., \& Norris, J. E. 2015, ARA\&A, 53, 631

Frischknecht, U., Hirschi, R., \& Thielemann, F.-K. 2012, A\&A, 538, L2

Frischknecht, U., Hirschi, R., Pignatari, M., et al. 2016, MNRAS, 456, 1803

Goriely, S., \& Siess, L. 2005, From Lithium to Uranium: Elemental Tracers of Early Cosmic Evolution, eds. V. Hill, P. Francois, \& F. Primas, IAU Symp., 228,451

Hansen, T., Andersen, J., Nordström, B., Buchhave, L., \& Beers, T. C. 2011, ApJ, 743, L1

Hansen, T. T., Hansen, C. J., Christlieb, N., et al. 2015a, ApJ, 807, 173

Hansen, T. T., Andersen, J., Nordström, B., et al. 2015b, A\&A, 583, A49 (Paper I)

Hansen, T. T., Andersen, J., Nordström, B., et al. 2016, A\&A, 586, A160 (Paper II)

Henden, A. A., Levine, S., Terrell, D., \& Welch, D. L. 2015, Am. Astron. Soc. Meet. Abstr., 225, 336.16

Hirschi, R. 2007, A\&A, 461, 571

Ivezic, Z., Beers, T. C., \& Juric, M. 2012, ARA\&A, 50, 251

Jorissen, A., Van Eck, S., Mayor, M., \& Udry, S. 1988, A\&A, 332, 877

Jorissen, A., Hansen, T., Van Eck, S., et al. 2016a, A\&A, 586, A159

Jorissen, A., Van Eck, S., Van Winkel, H., et al. 2016b, A\&A, 586, A158

Karakas, A. I. 2010, MNRAS, 403, 1413

Karakas, A. I, \& Lattanzio, J. C. 2014, PASA, 31, 30

Kennedy, C. R., Sivarani, T., \& Beers., T. C. 2011, AJ, 141, 102

Kopal, Z. 1959, Close Binary Systems, The International Astrophysics Series (London: Chapman Hall)

Lucatello, S, Gratton, R., Cohen, J. G., et al. 2003, AJ, 125, 875

Lucatello, S., Tsangarides, S. A., Beers, T. C., et al. 2005, ApJ, 625, 825

Lugaro, M., Karakas, A. I., Stancliffe, R. J., \& Rijs, C. 2012, ApJ, 747, 2

Maeder, A., Meynet, G., \& Chiappini, C. 2015, A\&A, 576, A56

Masseron, T., Johnson, J. A., Plez, B., et al. 2010, A\&A, 509, A93

Mathieu, R. D., Latham, D. W., \& Griffin, R. F. 1990, AJ, 100, 1899

McClure, R. D., \& Woodsworth, A. W. 1990, ApJ, 352, 709

Merle, T., Jorissen, A., Van Eck, S., Maseron, T., \& Van Winkel, H. 2016, A\&A, 586, A 151

Mermilliod, J.-C., Andersen, J., Latham, D. W., \& Mayor, M. 2007, A\&A, 473, 829

Meynet, G., Ekström, S., \& Maeder, A. 2006, A\&A, 447, 623

Mohamed, S., \& Podsiadlowski, P. 2007, ASP Conf. Ser. 372, eds. R. Napiwotzki, \& M. R. Burleigh, 397

Morbey, C. L., \& Griffin, R. F. 1987, ApJ, 317, 343

Nordström, B., Stefanik, R. P., Latham, D. W., \& Andersen, J. 1997, A\&AS, 126,21

Norris, J. E., Ryan, S. G., \& Beers, T. C. 1997, ApJ, 488, 350

Paczyński, B. 1965, Acta Astron., 15, 89

Paczyński, B. 1976, Structure and Evolution of Close Binary Systems, eds. P. Eggleton, S. Mitton, \& J. Whelan, IAU Symp., 73, 75

Pignatari, M., Gallino, R., Meynet, G., et al. 2008, ApJ, 687, L95

Placco, V. M., Kennedy, C. R., Rossi, S., et al. 2010, AJ, 139, 1051

Placco, V. M., Kennedy, C. R., Beers, T. C., et al. 2011, AJ, 142, 188

Placco, V. M., Frebel, A., Beers, T. C., et al. 2013, ApJ, 770, 104

Placco, V. M., Beers, T. C., Ivans, I. I., et al. 2015, ApJ, 812, 109

Preston, G. W., \& Sneden, C. 2000, AJ, 120, 1014

Richard, O., Michaud, G., Richer, J., et al. 2002, ApJ, 568, 979

Ricker, P. M., \& Taam, R. E. 2008, ApJ, 672, L41

Riebel, D., Meixner, M., Fraser, O., et al. 2010, ApJ, 723, 1195

Ryan, S. G., Aoki, W., Norris, J. E., \& Beers, T. C. 2005, ApJ, 635, 349

Spite, M., Caffau, E., Bonifacio, P., et al. 2013, A\&A, 552, A107

Stancliffe, R., \& Glebbeek, E. 2008, MNRAS, 389, 1828

Stancliffe, R., Glebbeek, E., Izzard, R. G., \& Pols., O. R. 2007, A\&A, 464, L57

Starkenburg, E., Shetrone, M. D., McConnachie, A. W., \& Venn, K. A. 2015, MNRAS, 441, 1217

Tsangarides, S. A., Ryan, S. G., \& Beers, T. C. 2003, ASP Conf. Ser. 304, eds. C. Charbonnel, D. Schaerer, \& G. Meynet, 133

Umeda, H. \& Nomoto, K. 2003, Nature, 422,871

Vassiliadis, E., \& Wood, P. R. 1993, ApJ, 413, 641 


\section{Appendix A: Radial velocities measured for the programme stars}

Table A.1. HE 0002-1037.

\begin{tabular}{lcc}
\hline \hline HJD & $\begin{array}{c}\mathrm{RV} \\
\mathrm{km} \mathrm{s}^{-1}\end{array}$ & $\begin{array}{c}\mathrm{RV}_{\text {err }} \\
\mathrm{km} \mathrm{s}^{-1}\end{array}$ \\
\hline 2456191.52615 & -36.229 & 0.045 \\
2456241.45815 & -33.883 & 0.184 \\
2456530.70894 & -24.359 & 0.040 \\
2456574.57567 & -27.581 & 0.044 \\
2456647.45601 & -35.052 & 0.081 \\
2456652.37785 & -35.635 & 0.053 \\
2456888.57469 & -38.668 & 0.061 \\
2456956.43565 & -34.844 & 0.076 \\
2457225.65699 & -22.941 & 0.065 \\
2457257.63749 & -23.761 & 0.055 \\
\hline
\end{tabular}

Table A.2. HE 0017+0055.

\begin{tabular}{lcc}
\hline \hline HJD & $\begin{array}{c}\mathrm{RV} \\
\mathrm{km} \mathrm{s}^{-1}\end{array}$ & $\begin{array}{c}\mathrm{RV}_{\mathrm{err}} \\
\mathrm{km} \mathrm{s}^{-1}\end{array}$ \\
\hline 2454314.67018 & -78.622 & 0.007 \\
2454338.64193 & -78.944 & 0.007 \\
2454373.62240 & -78.691 & 0.009 \\
2454396.53706 & -78.583 & 0.007 \\
2454406.59662 & -78.505 & 0.012 \\
2454480.38681 & -78.183 & 0.012 \\
2454793.48462 & -79.098 & 0.018 \\
2454820.33862 & -78.830 & 0.017 \\
2455059.73646 & -79.922 & 0.008 \\
2455149.47303 & -79.647 & 0.010 \\
2455207.34980 & -79.391 & 0.015 \\
2455415.60806 & -80.439 & 0.009 \\
2455439.59141 & -80.778 & 0.010 \\
2455503.40860 & -80.330 & 0.023 \\
2455738.73436 & -80.148 & 0.008 \\
2455776.68215 & -80.675 & 0.008 \\
2455821.57659 & -80.870 & 0.009 \\
2455944.32531 & -80.429 & 0.011 \\
2456139.71293 & -80.857 & 0.010 \\
2456241.39102 & -81.420 & 0.018 \\
2456545.62665 & -81.474 & 0.011 \\
2456652.41797 & -81.951 & 0.015 \\
2456686.32089 & -81.538 & 0.015 \\
2456840.71823 & -80.866 & 0.028 \\
2456888.54207 & -81.261 & 0.013 \\
2456917.59811 & -81.398 & 0.014 \\
2456987.38131 & -81.885 & 0.009 \\
2457257.57737 & -81.396 & 0.010 \\
\hline & &
\end{tabular}

Table A.3. HE 0111-1346.

\begin{tabular}{lcc}
\hline \hline HJD & $\begin{array}{c}\mathrm{RV} \\
\mathrm{km} \mathrm{s}^{-1}\end{array}$ & $\begin{array}{c}\mathrm{RV}_{\text {err }} \\
\mathrm{km} \mathrm{s}^{-1}\end{array}$ \\
\hline 2456213.65944 & +50.431 & 0.022 \\
2456531.67451 & +39.604 & 0.015 \\
2456534.72823 & +40.177 & 0.024 \\
2456574.62161 & +47.100 & 0.015 \\
2456579.62258 & +47.786 & 0.020 \\
2456652.40265 & +49.094 & 0.021 \\
2456893.65246 & +31.577 & 0.018 \\
2456917.61673 & +36.046 & 0.019 \\
2457257.65967 & +26.462 & 0.026 \\
\hline
\end{tabular}

Table A.4. HE 0151-0341.

\begin{tabular}{lcc}
\hline \hline HJD & $\begin{array}{c}\mathrm{RV} \\
\mathrm{km} \mathrm{s}^{-1}\end{array}$ & $\begin{array}{c}\mathrm{RV}_{\mathrm{err}} \\
\mathrm{km} \mathrm{s}^{-1}\end{array}$ \\
\hline 2456213.70382 & -37.298 & 0.070 \\
2456307.32814 & -25.696 & 0.064 \\
2456518.73462 & -47.217 & 0.041 \\
2456545.65829 & -42.753 & 0.047 \\
2456574.65686 & -36.767 & 0.030 \\
2456603.50990 & -31.030 & 0.042 \\
2456647.48211 & -25.892 & 0.065 \\
2456652.47616 & -25.703 & 0.072 \\
2456685.39340 & -26.739 & 0.065 \\
2456893.72033 & -44.812 & 0.051 \\
2457225.72100 & -48.629 & 0.065 \\
\hline
\end{tabular}

Table A.5. HE 0206-1916.

\begin{tabular}{lcc}
\hline \hline HJD & $\begin{array}{c}\mathrm{RV} \\
\mathrm{km} \mathrm{s}^{-1}\end{array}$ & $\begin{array}{c}\mathrm{RV}_{\text {err }} \\
\mathrm{km} \mathrm{s}^{-1}\end{array}$ \\
\hline 2456213.68126 & -199.551 & 0.047 \\
2456307.37509 & -199.446 & 0.052 \\
2456529.69669 & -199.422 & 0.024 \\
2456546.73298 & -199.723 & 0.060 \\
2456603.53471 & -199.651 & 0.041 \\
2456893.67497 & -199.471 & 0.033 \\
2456987.43608 & -199.626 & 0.061 \\
2457018.49730 & -199.580 & 0.073 \\
2457257.68120 & -199.356 & 0.037 \\
\hline
\end{tabular}

Table A.6. HE 0319-0215.

\begin{tabular}{lcc}
\hline \hline HJD & $\begin{array}{c}\mathrm{RV} \\
\mathrm{km} \mathrm{s}^{-1}\end{array}$ & $\begin{array}{c}\mathrm{RV}_{\text {err }} \\
\mathrm{km} \mathrm{s}^{-1}\end{array}$ \\
\hline 2454780.64369 & -229.933 & 0.029 \\
2455126.64403 & -227.058 & 0.024 \\
2455149.51200 & -226.974 & 0.030 \\
2455207.38599 & -226.673 & 0.017 \\
2455415.70963 & -224.274 & 0.023 \\
2455439.67958 & -224.389 & 0.019 \\
2455478.61056 & -224.219 & 0.022 \\
2455620.34838 & -223.797 & 0.026 \\
2455821.65016 & -222.893 & 0.012 \\
2455882.56766 & -223.207 & 0.020 \\
2455915.54130 & -223.575 & 0.117 \\
2456191.63308 & -223.890 & 0.017 \\
2456528.72760 & -226.084 & 0.028 \\
2456578.64692 & -226.565 & 0.018 \\
2456956.69477 & -229.280 & 0.085 \\
2456987.52074 & -229.692 & 0.063 \\
\hline
\end{tabular}


T. T. Hansen et al.: The role of binaries in the enrichment of the early Galactic halo. III.

Table A.7. HE 0430-1609.

\begin{tabular}{lcc}
\hline \hline HJD & $\begin{array}{c}\mathrm{RV} \\
\mathrm{km} \mathrm{s}^{-1}\end{array}$ & $\begin{array}{c}\mathrm{RV}_{\text {err }} \\
\mathrm{km} \mathrm{s}^{-1}\end{array}$ \\
\hline 2456209.69059 & +228.767 & 0.025 \\
2456214.65424 & +228.731 & 0.021 \\
2456529.71626 & +230.277 & 0.020 \\
2456545.73032 & +230.365 & 0.020 \\
2456574.68459 & +230.530 & 0.015 \\
2456685.46062 & +231.151 & 0.018 \\
2456722.36199 & +231.359 & 0.048 \\
2456893.69735 & +232.234 & 0.035 \\
2456917.63747 & +232.325 & 0.033 \\
2456956.76841 & +232.672 & 0.116 \\
2456986.65656 & +232.743 & 0.022 \\
2457076.35811 & +233.154 & 0.086 \\
2457092.34638 & +233.248 & 0.025 \\
2457110.35827 & +233.315 & 0.050 \\
2457257.71794 & +233.909 & 0.029 \\
2457393.47107 & +234.389 & 0.037 \\
\hline
\end{tabular}

Table A.8. HE 0441-0652.

\begin{tabular}{lcc}
\hline \hline HJD & $\begin{array}{c}\mathrm{RV} \\
\mathrm{km} \mathrm{s}^{-1}\end{array}$ & $\begin{array}{c}\mathrm{RV}_{\text {err }} \\
\mathrm{km} \mathrm{s}^{-1}\end{array}$ \\
\hline 2454705.73510 & -39.600 & 0.154 \\
2455149.58120 & -33.053 & 0.639 \\
2455176.49676 & -31.568 & 0.372 \\
2455207.53305 & -30.860 & 0.149 \\
2455439.70545 & -29.741 & 0.163 \\
2455478.64746 & -29.967 & 0.081 \\
2455531.57019 & -29.901 & 0.271 \\
2455821.67913 & -28.773 & 0.130 \\
2455915.58170 & -27.892 & 0.810 \\
2456005.34969 & -29.417 & 0.507 \\
2456191.66229 & -29.167 & 0.183 \\
2456214.67479 & -29.727 & 0.170 \\
2456574.73303 & -30.053 & 0.135 \\
2456685.48885 & -29.826 & 0.417 \\
2456987.46556 & -30.047 & 0.298 \\
2457076.43392 & -30.762 & 1.056 \\
\hline
\end{tabular}

Table A.9. HE 0507-1430.

\begin{tabular}{lcc}
\hline \hline HJD & $\begin{array}{c}\mathrm{RV} \\
\mathrm{km} \mathrm{s}^{-1}\end{array}$ & $\begin{array}{c}\mathrm{RV}_{\mathrm{err}} \\
\mathrm{km} \mathrm{s}^{-1}\end{array}$ \\
\hline 2455149.65141 & +53.744 & 0.017 \\
2455176.59963 & +53.614 & 0.027 \\
2455207.45478 & +51.698 & 0.016 \\
2455232.47668 & +48.880 & 0.016 \\
2455478.68157 & +40.340 & 0.029 \\
2455821.73671 & +32.139 & 0.018 \\
2455882.66528 & +34.746 & 0.032 \\
2455944.52431 & +43.196 & 0.044 \\
2455971.41362 & +47.094 & 0.037 \\
2456005.41341 & +51.227 & 0.077 \\
2456213.73374 & +36.148 & 0.030 \\
\hline
\end{tabular}

Table A.10. HE 0507-1653.

\begin{tabular}{lcc}
\hline \hline HJD & $\begin{array}{c}\mathrm{RV} \\
\mathrm{km} \mathrm{s}^{-1}\end{array}$ & $\begin{array}{c}\mathrm{RV}_{\text {err }} \\
\mathrm{km} \mathrm{s}^{-1}\end{array}$ \\
\hline 2454793.55000 & +346.064 & 0.053 \\
2454820.45944 & +349.034 & 0.025 \\
2455126.69729 & +342.750 & 0.015 \\
2455171.57052 & +343.843 & 0.014 \\
2455207.51291 & +347.058 & 0.018 \\
2455439.72402 & +349.472 & 0.014 \\
2455478.72231 & +345.614 & 0.052 \\
2455503.57008 & +343.789 & 0.046 \\
2455531.52335 & +342.751 & 0.030 \\
2455860.69191 & +347.747 & 0.015 \\
2455892.55739 & +344.930 & 0.117 \\
2456191.75734 & +354.904 & 0.012 \\
2456209.72120 & +353.449 & 0.021 \\
2456531.71198 & +356.770 & 0.018 \\
2456917.65964 & +356.020 & 0.025 \\
\hline
\end{tabular}

Table A.11. HE 0854+0151

\begin{tabular}{lcc}
\hline \hline HJD & $\begin{array}{c}\mathrm{RV} \\
\mathrm{km} \mathrm{s}^{-1}\end{array}$ & $\begin{array}{c}\mathrm{RV}_{\text {err }} \\
\mathrm{km} \mathrm{s}^{-1}\end{array}$ \\
\hline 2454516.50743 & +135.575 & 0.033 \\
2454780.70470 & +144.261 & 0.072 \\
2454930.38332 & +130.585 & 0.034 \\
2454964.39430 & +124.541 & 0.054 \\
2455149.73265 & +141.161 & 0.032 \\
2455171.71750 & +144.308 & 0.024 \\
2455174.67146 & +144.662 & 0.041 \\
2455232.52829 & +145.394 & 0.025 \\
2455531.69023 & +139.809 & 0.040 \\
2455620.45671 & +145.626 & 0.041 \\
2455662.42371 & +140.074 & 0.028 \\
2455822.74479 & +121.901 & 0.112 \\
2455915.75991 & +138.642 & 0.129 \\
2456005.48541 & +145.867 & 0.061 \\
2456273.71422 & +132.054 & 0.062 \\
\hline
\end{tabular}

Table A.12. HE 0959-1424.

\begin{tabular}{lcc}
\hline \hline HJD & $\begin{array}{c}\mathrm{RV} \\
\mathrm{km} \mathrm{s}^{-1}\end{array}$ & $\begin{array}{c}\mathrm{RV}_{\text {err }} \\
\mathrm{km} \mathrm{s}^{-1}\end{array}$ \\
\hline 2454406.77276 & +344.076 & 0.171 \\
2454793.71150 & +344.176 & 0.409 \\
2455149.76314 & +343.803 & 0.156 \\
2455174.72219 & +343.757 & 0.181 \\
2455207.58849 & +343.775 & 0.145 \\
2455310.37042 & +343.598 & 0.137 \\
2455544.72364 & +343.630 & 0.204 \\
2455620.54421 & +343.708 & 0.140 \\
2455662.38833 & +343.659 & 0.128 \\
2455944.56413 & +343.632 & 0.246 \\
2456005.51849 & +343.377 & 0.283 \\
2456033.36757 & +343.459 & 0.357 \\
2456399.38501 & +343.025 & 0.248 \\
2456722.52147 & +342.309 & 0.519 \\
2456756.38762 & +342.602 & 0.268 \\
2456796.37413 & +342.613 & 0.174 \\
2457142.38500 & +341.818 & 0.273 \\
\hline
\end{tabular}


Table A.13. HE 1045+0226.

\begin{tabular}{lcc}
\hline \hline HJD & $\begin{array}{c}\mathrm{RV} \\
\mathrm{km} \mathrm{s}^{-1}\end{array}$ & $\begin{array}{c}\mathrm{RV}_{\text {err }} \\
\mathrm{km} \mathrm{s}^{-1}\end{array}$ \\
\hline 2456307.69698 & +131.672 & 0.228 \\
2456399.43421 & +131.652 & 0.099 \\
2456652.61751 & +131.564 & 0.104 \\
2456712.67531 & +131.685 & 0.364 \\
2457076.61932 & +131.460 & 0.399 \\
2457110.44369 & +130.954 & 0.178 \\
\hline
\end{tabular}

Table A.14. HE 1046-1352.

\begin{tabular}{lcc}
\hline \hline HJD & $\begin{array}{c}\mathrm{RV} \\
\mathrm{km} \mathrm{s}^{-1}\end{array}$ & $\begin{array}{c}\mathrm{RV}_{\text {err }} \\
\mathrm{km} \mathrm{s}^{-1}\end{array}$ \\
\hline 2454909.61389 & +94.395 & 0.795 \\
2454930.45563 & +97.658 & 0.147 \\
2454964.43469 & +47.693 & 1.670 \\
2455174.74220 & +105.913 & 0.851 \\
2455232.57334 & +96.330 & 0.147 \\
2455310.39271 & +73.223 & 0.859 \\
2455344.39166 & +48.887 & 0.278 \\
2455554.78013 & +94.567 & 0.543 \\
2455620.51605 & +97.700 & 0.553 \\
2456006.50365 & +71.838 & 0.545 \\
2456033.41230 & +51.174 & 1.102 \\
2456721.61189 & +74.268 & 1.555 \\
\hline
\end{tabular}

Table A.15. CS 30301-015.

\begin{tabular}{lcc}
\hline \hline HJD & $\begin{array}{c}\mathrm{RV} \\
\mathrm{km} \mathrm{s}^{-1}\end{array}$ & $\begin{array}{c}\mathrm{RV}_{\text {err }} \\
\mathrm{km} \mathrm{s}^{-1}\end{array}$ \\
\hline 2454254.62579 & +86.747 & 0.094 \\
2454314.47889 & +86.510 & 0.081 \\
2454480.79001 & +86.638 & 0.054 \\
2454625.52411 & +86.607 & 0.049 \\
2454909.59318 & +86.585 & 0.078 \\
2454930.71839 & +86.636 & 0.066 \\
2454951.73429 & +86.674 & 0.094 \\
2454987.49075 & +86.577 & 0.048 \\
2455059.37482 & +86.462 & 0.055 \\
2455344.59436 & +86.562 & 0.048 \\
2455415.40116 & +86.663 & 0.058 \\
2455439.37958 & +86.510 & 0.056 \\
2455704.56070 & +86.620 & 0.065 \\
2455776.46938 & +86.611 & 0.064 \\
2456005.71388 & +86.564 & 0.134 \\
2456033.63882 & +86.633 & 0.058 \\
2456078.51945 & +86.566 & 0.061 \\
2456488.39989 & +86.763 & 0.044 \\
\hline
\end{tabular}

Table A.16. HE 1523-1155.

\begin{tabular}{lcc}
\hline \hline HJD & $\begin{array}{c}\mathrm{RV} \\
\mathrm{km} \mathrm{s}^{-1}\end{array}$ & $\begin{array}{c}\mathrm{RV}_{\text {err }} \\
\mathrm{km} \mathrm{s}^{-1}\end{array}$ \\
\hline 2456756.69769 & -48.312 & 0.034 \\
2456796.67508 & -46.377 & 0.051 \\
2456888.38390 & -40.622 & 0.040 \\
2457110.72622 & -46.063 & 0.069 \\
2457142.65599 & -44.028 & 0.037 \\
2457168.61410 & -42.363 & 0.026 \\
2457225.44781 & -39.130 & 0.042 \\
2457239.39307 & -38.552 & 0.020 \\
2457258.36919 & -38.016 & 0.042 \\
\hline
\end{tabular}

Table A.17. HE 2201-0345.

\begin{tabular}{lcc}
\hline \hline HJD & $\begin{array}{c}\mathrm{RV} \\
\mathrm{km} \mathrm{s}^{-1}\end{array}$ & $\begin{array}{c}\mathrm{RV}_{\text {err }} \\
\mathrm{km} \mathrm{s}^{-1}\end{array}$ \\
\hline 2454314.59436 & -54.804 & 0.053 \\
2454338.51241 & -54.763 & 0.098 \\
2454373.44648 & -54.627 & 0.078 \\
2454665.70652 & -53.021 & 0.093 \\
2454964.70705 & -50.946 & 0.142 \\
2455009.67131 & -50.441 & 0.093 \\
2455059.48210 & -50.167 & 0.071 \\
2455126.49880 & -49.927 & 0.067 \\
2455344.66126 & -51.510 & 0.053 \\
2455415.47351 & -52.802 & 0.062 \\
2455439.41067 & -53.130 & 0.051 \\
2455503.33221 & -54.321 & 0.155 \\
2455531.36578 & -54.418 & 0.185 \\
2455704.66231 & -56.625 & 0.042 \\
2455776.49350 & -57.152 & 0.086 \\
2455882.30261 & -58.114 & 0.092 \\
2455898.30389 & -58.260 & 0.091 \\
2456033.73856 & -58.053 & 0.236 \\
2456078.70413 & -58.791 & 0.096 \\
2456139.62139 & -58.890 & 0.102 \\
2456518.50427 & -59.730 & 0.532 \\
2456530.61456 & -59.760 & 0.062 \\
2456603.44706 & -59.703 & 0.121 \\
2456886.51808 & -59.808 & 0.085 \\
2456956.46129 & -60.095 & 0.197 \\
2457192.70683 & -60.170 & 0.059 \\
2457257.54595 & -60.008 & 0.100 \\
\hline
\end{tabular}

Table A.18. HE 2312-0758.

\begin{tabular}{lcc}
\hline \hline HJD & $\begin{array}{c}\mathrm{RV} \\
\mathrm{km} \mathrm{s}^{-1}\end{array}$ & $\begin{array}{c}\mathrm{RV}_{\text {err }} \\
\mathrm{km} \mathrm{s}^{-1}\end{array}$ \\
\hline 2456191.43803 & +38.553 & 0.050 \\
2456213.45637 & +38.391 & 0.054 \\
2456488.71018 & +34.121 & 0.045 \\
2456518.67707 & +33.749 & 0.051 \\
2456528.66775 & +33.640 & 0.063 \\
2456545.61190 & +33.228 & 0.046 \\
2456887.52097 & +30.174 & 0.067 \\
2456917.53578 & +30.435 & 0.073 \\
2456956.48012 & +30.255 & 0.074 \\
2457225.62292 & +30.102 & 0.062 \\
2457257.56346 & +30.139 & 0.048 \\
\hline
\end{tabular}

Table A.19. HE 2330-0555.

\begin{tabular}{lcc}
\hline \hline HJD & $\begin{array}{c}\mathrm{RV} \\
\mathrm{km} \mathrm{s}^{-1}\end{array}$ & $\begin{array}{c}\mathrm{RV}_{\mathrm{err}} \\
\mathrm{km} \mathrm{s}^{-1}\end{array}$ \\
\hline 2454314.711836 & -234.968 & 0.046 \\
2454373.550888 & -235.089 & 0.052 \\
2455059.605701 & -235.218 & 0.054 \\
2455080.608758 & -235.039 & 0.108 \\
2455126.539073 & -235.182 & 0.059 \\
2455176.414354 & -235.118 & 0.142 \\
2455415.633807 & -235.179 & 0.052 \\
2455439.497335 & -235.104 & 0.060 \\
2455478.573649 & -235.114 & 0.078 \\
2455531.439197 & -235.126 & 0.094 \\
2455776.707594 & -235.231 & 0.070 \\
2455796.607358 & -235.370 & 0.091 \\
2455882.421279 & -235.048 & 0.045 \\
2456141.696801 & -234.927 & 0.092 \\
2456241.363645 & -234.912 & 0.212 \\
2456530.642253 & -235.139 & 0.066 \\
2456887.546840 & -235.386 & 0.094 \\
\hline & &
\end{tabular}


T. T. Hansen et al.: The role of binaries in the enrichment of the early Galactic halo. III.

Table A.20. HE 0039-2635.

\begin{tabular}{lcc}
\hline \hline HJD & $\begin{array}{c}\mathrm{RV} \\
\mathrm{km} \mathrm{s}^{-1}\end{array}$ & $\begin{array}{c}\mathrm{RV}_{\text {err }} \\
\mathrm{km} \mathrm{s}^{-1}\end{array}$ \\
\hline 2456241.41669 & -52.078 & 0.023 \\
2456518.71254 & -43.400 & 0.023 \\
\hline
\end{tabular}

Table A.21. HE 1031-0020.

\begin{tabular}{lcc}
\hline \hline HJD & $\begin{array}{c}\mathrm{RV} \\
\mathrm{km} \mathrm{s}^{-1}\end{array}$ & $\begin{array}{c}\mathrm{RV}_{\text {err }} \\
\mathrm{km} \mathrm{s}^{-1}\end{array}$ \\
\hline 2454219.42938 & +69.718 & 0.103 \\
2454254.44108 & +69.319 & 0.152 \\
2454406.74096 & +69.519 & 0.119 \\
2454459.64146 & +69.839 & 0.141 \\
2454464.63446 & +69.944 & 0.086 \\
2454481.61235 & +69.524 & 0.145 \\
2454516.55332 & +69.634 & 0.096 \\
2454793.76871 & +69.565 & 0.136 \\
2454909.51656 & +69.473 & 0.124 \\
2454930.53075 & +69.308 & 0.067 \\
2455174.70264 & +69.198 & 0.095 \\
2455207.60630 & +69.142 & 0.075 \\
2455544.74047 & +68.903 & 0.114 \\
2455620.49236 & +68.592 & 0.096 \\
2455915.79512 & +68.460 & 0.222 \\
2455944.58281 & +67.917 & 0.197 \\
2456033.38640 & +68.077 & 0.098 \\
2456241.74480 & +67.609 & 0.138 \\
2456399.40659 & +67.486 & 0.120 \\
2456722.48940 & +66.819 & 0.149 \\
2457110.39343 & +66.361 & 0.227 \\
2457142.44270 & +66.111 & 0.106 \\
\hline
\end{tabular}

Table A.22. LP 625-44.

\begin{tabular}{lcc}
\hline \hline HJD & $\begin{array}{c}\mathrm{RV} \\
\mathrm{km} \mathrm{s}^{-1}\end{array}$ & $\begin{array}{c}\mathrm{RV}_{\mathrm{err}} \\
\mathrm{km} \mathrm{s}^{-1}\end{array}$ \\
\hline 2454219.72236 & +38.955 & 0.039 \\
2454254.65878 & +38.870 & 0.111 \\
2454285.56286 & +39.027 & 0.060 \\
2454314.49424 & +38.911 & 0.041 \\
2454338.41720 & +38.770 & 0.047 \\
2454373.35738 & +38.773 & 0.051 \\
2454625.62936 & +38.051 & 0.038 \\
2454665.57095 & +38.081 & 0.093 \\
2454930.74622 & +37.306 & 0.046 \\
2454964.69845 & +37.168 & 0.059 \\
2454987.52690 & +37.153 & 0.036 \\
2455059.43020 & +36.853 & 0.075 \\
2455344.60471 & +36.007 & 0.060 \\
2455415.37378 & +35.652 & 0.057 \\
2455439.39220 & +35.608 & 0.057 \\
2455620.75079 & +34.869 & 0.059 \\
2455704.58037 & +34.615 & 0.062 \\
2455776.41360 & +34.301 & 0.048 \\
2456005.74059 & +33.446 & 0.086 \\
2456033.68185 & +33.154 & 0.106 \\
2456062.71485 & +33.086 & 0.091 \\
2456078.55749 & +32.913 & 0.069 \\
2456488.41291 & +30.863 & 0.052 \\
2456574.33217 & +30.479 & 0.037 \\
2456579.32582 & +30.406 & 0.081 \\
2456756.74336 & +29.588 & 0.167 \\
2456796.71349 & +29.225 & 0.068 \\
2456887.37991 & +28.872 & 0.056 \\
\hline & & \\
\hline
\end{tabular}


A\&A 588, A3 (2016)

\section{Appendix B: Literature data for the single programme stars}

Table B.1. Mean heliocentric radial velocities from the literature and total time span covered for the single stars.

\begin{tabular}{lrrrll}
\hline \hline Stellar ID & $\begin{array}{r}\Delta T \text { Total } \\
(\text { days })\end{array}$ & $\begin{array}{r}\text { Mean RV (this work) } \\
\left(\mathrm{km} \mathrm{s}^{-1}\right)\end{array}$ & $\begin{array}{r}\text { Mean RV } \\
\left(\mathrm{km} \mathrm{s}^{-1}\right)\end{array}$ & N \\
\hline HE 0206-1916 & 4445 & -199.509 & -200.0 & 1 & Aoki et al. (2007) \\
\hline HE 1045+0226 & 1868 & +131.498 & +131.2 & 1 & Cohen et al. (2013) \\
\hline HE 2330-0555 & 4314 & -235.124 & -235.0 & 1 & Aoki et al. (2007) \\
\hline CS 30301-015 & 4352 & +86.61 & +85.5 & 1 & Tsangarides et al. (2003) \\
& & & +86.5 & 1 & Aoki et al. (2002b) \\
& & & +85.5 & 2 & Lucatello et al. (2005) \\
\hline
\end{tabular}

